\title{
Society of Vascular and Interventional Neurology (SVIN) Stroke Interventional Laboratory Consensus (SILC) Criteria: A 7M Management Approach to Developing a Stroke Interventional Laboratory in the Era of Stroke Thrombectomy for Large Vessel Occlusions
}

\author{
Tanzila Shams $^{a} \quad$ Osama Zaidat $^{b}$ Dileep Yavagalc ${ }^{c}$ Andrew Xavier ${ }^{d}$ \\ Tudor Jovin $^{\mathrm{e}}$ Vallabh Janardhan ${ }^{\mathrm{a}}$ \\ ${ }^{a}$ Texas Stroke Institute, HCA North Texas Division, Dallas-Fort Worth, Tex., bercy \\ Neuroscience and Stroke Center, Toledo, Ohio, 'Jackson Memorial Hospital, University of \\ Miami Health System, Miami, Fla., ${ }^{d}$ Detroit Medical Center, Wayne State University, \\ Detroit, Mich., and e UPMC Stroke Institute, University of Pittsburgh Medical Center, \\ Pittsburg, Pa., USA
}

\section{Key Words}

Acute ischemic stroke - Large vessel occlusion - Endovascular therapy - Stroke intervention . Quality standards

\begin{abstract}
Brain attack care is rapidly evolving with cutting-edge stroke interventions similar to the growth of heart attack care with cardiac interventions in the last two decades. As the field of stroke intervention is growing exponentially globally, there is clearly an unmet need to standardize stroke interventional laboratories for safe, effective, and timely stroke care. Towards this goal, the Society of Vascular and Interventional Neurology (SVIN) Writing Committee has developed the Stroke Interventional Laboratory Consensus (SILC) criteria using a 7M management approach for the development and standardization of each stroke interventional laboratory within stroke centers. The SILC criteria include: (1) manpower: personnel including roles of medical and administrative directors, attending physicians, fellows, physician extenders, and all the key stakeholders in the stroke chain of survival; (2) machines: resources needed in terms of physical facilities, and angiography equipment; (3) materials: medical device inventory, medications, and angiography supplies; (4) methods: standardized protocols for stroke
\end{abstract}


workflow optimization; (5) metrics (volume): existing credentialing criteria for facilities and stroke interventionalists; (6) metrics (quality): benchmarks for quality assurance; (7) metrics (safety): radiation and procedural safety practices.

(C) 2016 S. Karger AG, Basel

\section{Introduction}

\section{Organization of Committee and Evidence Review}

The Society of Vascular and Interventional Neurology (SVIN) formed a Task Force and a Writing Committee to develop the Stroke Interventional Laboratory Consensus Criteria (SILC), which will help standardize the quality of stroke interventional laboratories worldwide. Academic (university hospital) and real-world (community hospital) experts are both represented in the SILC Taskforce and Writing Committee. An expert panel of official reviewers nominated by the SVIN Writing committee has reviewed this document. This document will delineate the recommended consensus criteria to help standardize all new and existing stroke interventional laboratories worldwide, and shall be considered current until the SVIN SILC Taskforce and Writing Committee has further updates.

\section{Purpose of This Expert Consensus Document}

The natural history of large vessel occlusion (LVO) acute ischemic stroke (AIS) has shown that brain attacks due to LVOs are a malignant disease with nearly $80 \%$ of the patients being dead or disabled for life, and is similar to the impact of ST-elevation myocardial infarction (STEMI) for patients with heart attacks [1]. Recent landmark trials have proven the safety and efficacy of endovascular therapy for brain attacks due to LVOs, similar to the landmark trials for primary coronary intervention (PCI) for heart attacks due to STEMIs decades ago [2, 3]. There is clearly an unmet need to understand how to reorganize stroke systems of care and develop quality interventional laboratories such that the LVO AIS patients are rapidly triaged to a Stroke Interventional Laboratory capable hospital and receive high-quality endovascular stroke therapy, similar to PCI-capable hospitals for STEMIs [4].

National and global systemic efforts in the uniform organization, accreditation, and certification of stroke intervention practices are more precedent than ever. Multiple societies and organizations including National Institute of Neurological Disorders and Stroke (NINDS) Brain Attack Coalition, American Heart Association/American Stroke Association (AHA/ASA), and certifying bodies including The Joint Commission (TJC) [5] Det Norske Veritas (DNV) [6], and Hospital Facilities Accreditation Program (HFAP) [6], endorse the systematic organization of stroke care operations, both ischemic and hemorrhagic, at a national level, and as a result the necessity and volume of stroke interventional procedures have risen uniformly across the United States [7, 8]. Due to increasing evidence through randomized controlled trials (fig. 1) towards the role of catheter-based diagnostic and therapeutic procedures in management of AIS within the last decade, stroke intervention practices have become commonplace. Further, in efforts to streamline resource utilization within hospital systems, a significant volume of nonemergent elective angiographic procedures are increasingly performed on an outpatient basis.

The setting of stroke interventions has evolved from the traditional exclusively academic tertiary hospital in the 1990s to the existing tiered systems of stroke operations across the United States. Currently, level 3 (acute stroke ready), level 2 (primary stroke centers), and level 1 (comprehensive stroke centers) are each tiered based on the ability to intake strokes, administer intravenous recombinant tissue plasminogen activator (IV t-PA), advanced monitoring, 24/7 access to a neurocritical care unit (Neuro ICU), neurosurgical, and stroke intervention services. Further, the certification bodies individually evaluate the institution's ability 
Shams et al.: Society of Vascular and Interventional Neurology (SVIN) Stroke Interventional Laboratory Consensus (SILC) Criteria

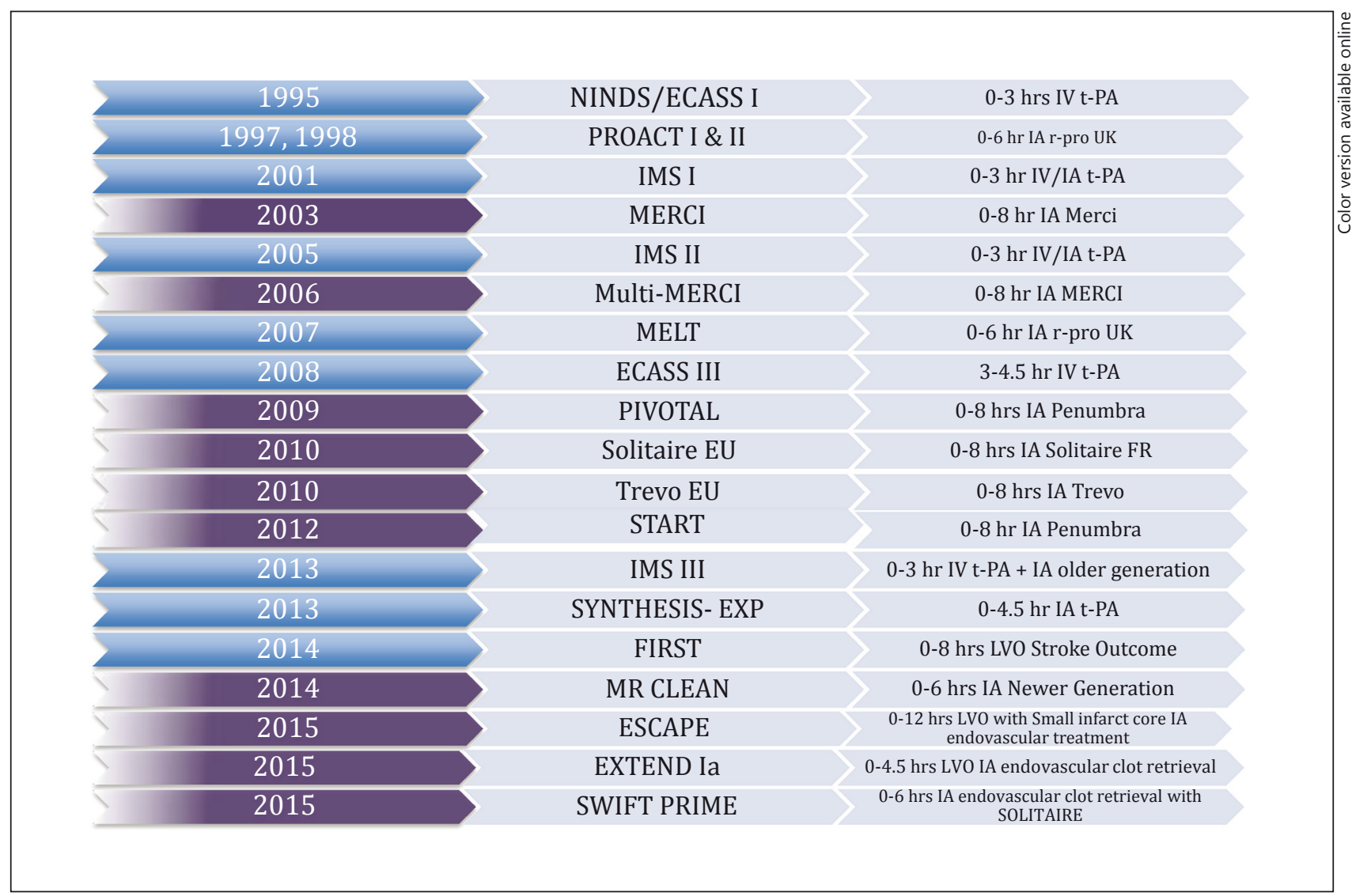

Fig. 1. Landmark stroke interventional trials. IA = Intra-arterial. Blue color indicates MEDICAL therapeutics trials. Purple color indicates device-based stroke interventional trials. Colors refer to the online version only.

to provide carotid stenting procedures and the capabilities of the rehabilitation center to provide optimal care in stroke-specific convalescence (fig. 2). Although this tiered system of stroke centers have been successfully established with improved outcomes in the medical treatment of strokes, little emphasis is placed on the development and operations of the stroke interventional lab within a stroke center. The various key parameters (technical, safety, equipment, and case volume parameters among others) essential for an angiographic stroke interventional laboratory to operate at a level of excellence in stroke care is poorly defined. With goals to deliver evidence-based, resource-efficient measures to improve patient outcomes, the SVIN Writing Committee proposes the standardization of stroke interventional laboratories to further optimize stroke operations systems globally.

Tiered Health Systems and Prehospital Triaging for Time-Sensitive Diseases

To best prepare for a new national standard of stroke care and provide optimal, timely stroke interventions for AIS patients, the current collective sentiment among stroke interventionalists reflects a strong call for universal standardization across all stroke interventional laboratories. Several models of structuring have been proposed in terms of facility-based designations. Quality metrics per segment of care delivery including prehospital assessments [9], emergency room events at drip-and-ship hospitals to optimize 'door in door out' times, and stroke interventional laboratory parameters to optimize 'door to perfusion' times are essential tools in guidance towards improvement of efficiency and outcomes. 
Fig. 2. Current stroke services recognized nationally within the stroke systems of care.
C) 2016 S. Karger AG, Basel www.karger.com/ine

Shams et al.: Society of Vascular and Interventional Neurology (SVIN) Stroke Interventional Laboratory Consensus (SILC) Criteria

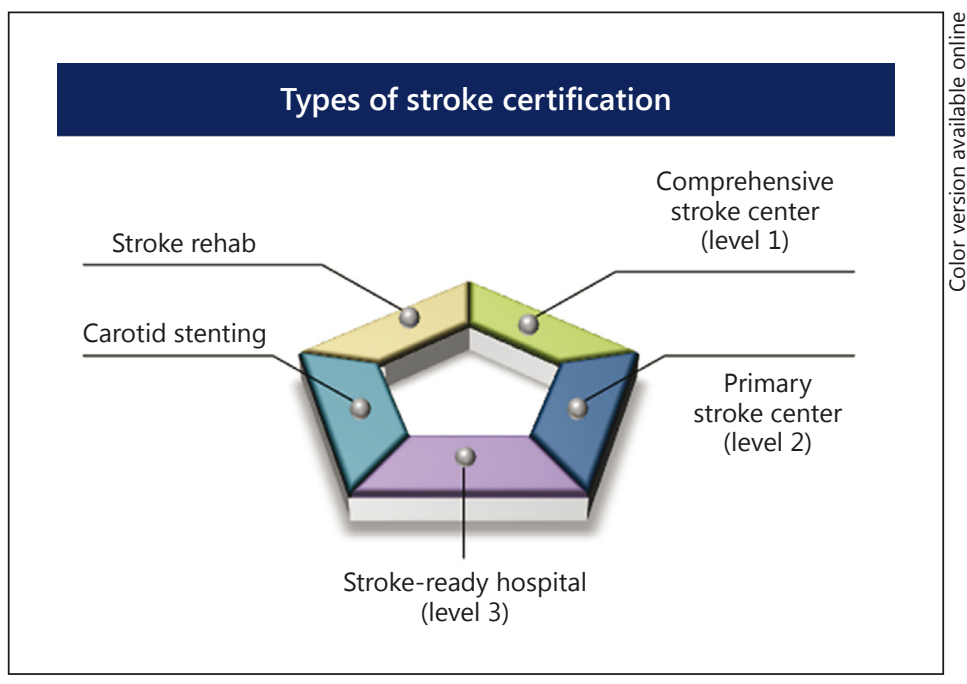

Lessons Learned from Trauma Systems of Care

The American Trauma Society (ATS) have successfully implemented a designated 5-level national trauma systems of operation, utilizing the collective resources of prehospital emergency services and emergency room personnel with the mission towards the 'the right care, to the right place, at the right time' [10-12]. Similar to the national trauma model, access to stroke interventions may also be structured according to facility capacities, levels of services, strategically targeted to at risk populations with the aim to provide a stroke patient with access to a designated stroke center, by either ambulance or helicopter, within 60 min of onset of symptoms, termed the 'golden hour of thrombolysis' [13].

Lessons Learned from Cardiac Systems of Care

Another treatment-centric structural model implemented by the AHA Mission Lifeline [14] is one specific to improving outcomes in myocardial infarction and time-targeted reperfusion rates, also known as ST-segment elevation (STEMI) systems of care. In efforts to achieve optimal myocardial reperfusion rates within the community, hospitals are designated as STEMI referring centers versus STEMI receiving centers, otherwise called PCI-capable hospitals $[15,16]$. STEMI referring centers are capable of cardiac catheterizations and pharmacological-based fibrinolytic treatments of acute heart attacks. STEMI receiving centers otherwise known as PCI-capable facilities are equipped to perform 24/7 mechanical percutaneous coronary intervention and are staffed by appropriately trained and experienced interventional cardiologists. This national effort in reorganization of heart attack care has improved national mortality and timely reperfusion rates [17]. Brain attack care and stroke systems may successfully emulate such a treatment-centric system by triaging all strokes eligible for stroke intervention as stroke receiving (24/7 drug delivery + device capable; stroke interventional laboratory capable facility) versus stroke referring facilities (only 24/7 drug delivery capable). By empowering emergency medical services (EMS) to appropriately triage stroke patients to receiving versus referring facilities according to availability of $24 / 7$ endovascular stroke intervention, stroke reperfusion rates may be further optimized, thus reducing morbidity and mortality.

Lessons Learned from Stroke Systems of Care

The existing model of stroke systems of care, chaired by the AHA/ASA [3], and initiatives such as Target: Stroke [18] have significantly improved national outcomes in stroke patients. 
Shams et al.: Society of Vascular and Interventional Neurology (SVIN) Stroke Interventional Laboratory Consensus (SILC) Criteria

7M approach - a lean operations method to stroke intervention

\section{Manpower}

\section{Machines}

Materials

Methods

Metrics-volume

Metrics-quality

Metrics-safety
Essential personnel; all key stakeholders for chain of survival in stroke care

Resources necessary in physical facilities and angiographic equipment

Medical device inventory, angiographic supplies, and other medical supplies

Standardized protocols for stroke workflow optimization

Credentialling criteria for facilities and interventionalists

Benchmarks for quality assurance and continued improvements

Radiation and procedural safety practices

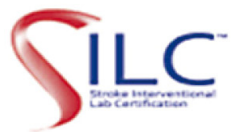

Fig. 3. The 7M management approach to developing a stroke interventional lab.

However, primary focus remains on stabilization and pharmacological IV t-PA treatment of acute strokes with little focus towards achieving excellence in stroke interventions. Landmark trials in intra-arterial treatments of AIS such as MR CLEAN [19], ESCAPE [20], EXTEND IA [21], SWIFT PRIME [22], and REVASCAT [23], have demonstrated the efficacy of mechanical thrombectomy in AIS, and there is a clear unmet need to define access and delivery of stroke intervention to appropriate LVO AIS patients. Restructuring of the stroke system to incorporate the SILC criteria can potentially further improve mortality and disabilities in stroke.

Proposed Future Tiered System for EMS Triage of Stroke

The future success of Brain Attack Care with stroke intervention and intra-arterial therapy lies in a lean supply chain method of operations that identifies efficiencies (value added) operations and wastes (non-value added) operations (fig. 3). Further, brain attack care must advance beyond prehospital notification to facilities and mirror the organization of heart attack care, designated as referring versus receiving hospitals (fig. 4). In recognition of 'the golden hour' of stroke, time to IV t-PA target initiatives, such as 'door to needle' times, have successfully improved IV t-PA administration rates within $60 \mathrm{~min}$ [18]; however, similar initiatives are currently lacking in time to intra-arterial treatments, such as 'door to perfusion' times for brain attacks similar to 'door to balloon' for heart attacks, and clear benchmarks have been discussed but not clearly established [23]. Effective stroke interventions to appropriate patients can be achieved with several key factors in place simultaneously: (a) clear designation of receiving facilities capable of stroke intervention for brain attack care (stroke interventional lab capable facilities); (b) initiation of national benchmarks targeted to reduce 'first medical contact to door' and 'door to perfusion' times, along with task forces charged 
Fig. 4. Parallels in future directions of brain attack care and heart attack care.

\section{EMS Triage for Brain attacks mirror Heart attacks}

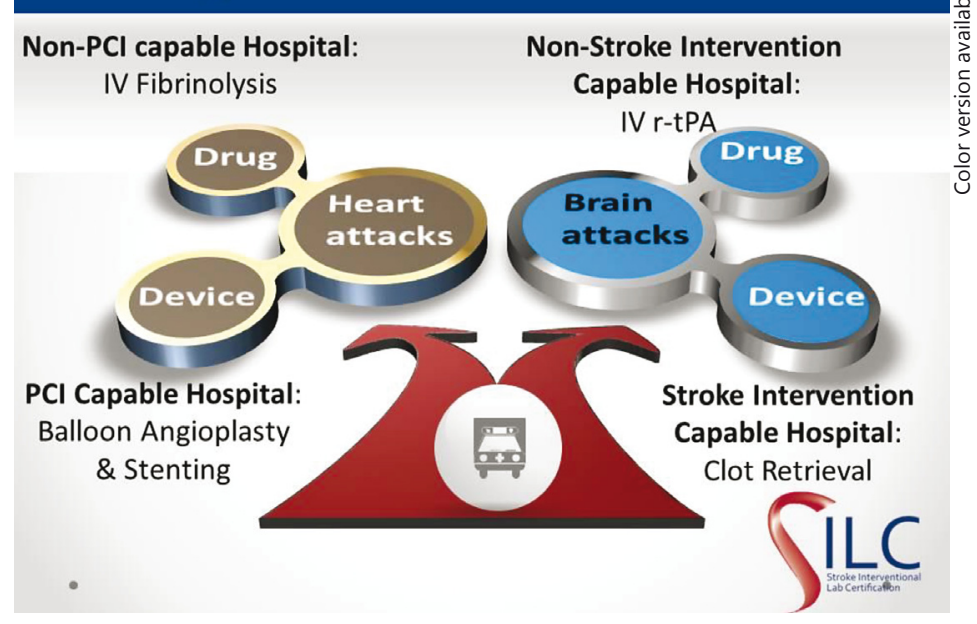

with accomplishing such benchmarks; (c) enforcing standards for stroke interventional laboratories that independently comply with quality standards of SILC criteria. Lean supply chain method of operations can therefore be applied to brain attack care in all 3 phases of operations - prehospital, emergency department (ED), and stroke interventional laboratory, as a whole system, with successful elimination of delays in treatment.

\section{Quality Metrics}

In efforts to improve outcomes in AIS, joint initiatives from AHA/ASA and NINDS have established methods for the best medical management and timely administration of IV t-PA. In the current environment of recently positive endovascular stroke clinical trials [19-23] that demonstrate improved outcomes in stroke patients that undergo timely catheter-based interventions, the burgeoning necessity for encompassing similar methods for stroke interventional treatments is imminent. Further, recent spotlight has been placed on pre-arrival interventions within ultra-early time windows such as early recognition and assessment of stroke severity by EMS to optimize triage of LVO AIS to a stroke interventional laboratory capable facility [24-26], administration of neurotherapeutics [27, 28], antihypertensives [29], and thrombolytics [30]. Improvement for emergency acute stroke treatments is currently an ongoing process, and focus should be placed on all systems of care, including initial contact by EMS, prehospital stroke severity assessment to rapidly identify LVO AIS, stroke interventions, and the complex sequence of events that lead up to the timely delivery of stroke intervention.

Currently, multi-society quality improvement guidelines for intra-arterial catheterdirected treatment of AIS recommend the collection of certain key metrics in order to improve and ensure efficiency within stroke intervention procedures [31]. Essential data points include door to imaging, door to puncture, puncture to recanalization, post-procedural imaging, rates of symptomatic ICH, and clinical outcomes. These are recommended at a minimum for any program providing stroke interventions, and serve as tools to improve national stroke outcomes. SVIN Writing Committee recommends that segments of stroke care delivery should be further compartmentalized as (1) prehospital (EMS) benchmarks, (2) in hospital (emergency room) metrics, and (3) laboratory (stroke interventional) metrics, in order to efficiently deliver stroke interventional services with good patient outcomes, and identify opportunities for future improvements. Stroke intervention laboratories may also be further certified according to initial and maintenance of procedure volumes including diag- 
nostic cerebral angiograms, acute stroke interventions including mechanical thrombectomies, endovascular treatment of cerebral aneurysms (coils, intra-aneurysm fillers, stents, or flow diverters), embolizations, and carotid artery stents. The future of national initiatives in stroke systems of care must inevitably cast a wider focus to incorporate all aspects of stroke care including ultra-early prehospital assessments at the onset of symptoms, efficient emergency room care with speedy door in door out parameters, and interventional laboratory parameters for intra-arterial therapies in AIS. Stroke interventional laboratories ought to be prepared in capacity and framework in advance.

Complications of stroke interventional procedures include intracerebral hemorrhage, an increased propensity for transient neurological deficits and ischemic strokes by either embolic or hemodynamic mechanisms. These life-threatening complications carry grave implications in complexity of management and frequently encounter unique ethical dilemmas in goals of care, and are thus most likely the reason, along with poor community understanding of the field, for reluctance in a referring physician to recommend such procedures. Risks of adverse events and complications from diagnostic cerebral angiograms, although not null [32], have declined significantly and therefore may safely be performed within a laboratory facility equipped with appropriately trained personnel, technology, and protocols. The incidence of adverse events after stroke interventional procedures of the cerebrovascular system, including intra- and extracranial, anterior and posterior circulations are largely underreported, but according to literature review, vary widely from 12 to $20 \%$ [33, 34]. For the safety of patients and the stroke community at large, any stroke interventional procedure for any cerebrovascular indication must be performed in the setting of full access to support services including neurology, neurosurgery, and neurointensive care at a facility that meets the SILC criteria to maintain the highest quality standards.

Radiographic equipment, imaging, and novel therapeutic devices have also rapidly improved since the 1970s [35], and are credited with significant impact on modeling technical parameters of a stroke interventional laboratory. Digital images and communications in medicine (DICOM) standards and digital subtraction angiography (DSA) have basically eliminated the utility of cinefilms [36], which require cumbersome manual techniques and result in subpar imprecise image quality in comparison to DSA. Full utilization of internet resources, electronic medical records, and cloud-based technology are imminent. 'Ad hoc' catheter revascularization and other stroke interventions are increasingly performed immediately following the diagnostic angiographic component of the procedure. The scope of stroke interventional diagnostics in specialty populations such as pediatrics and pregnancy have already been established, but continue to grow and evolve with regard to therapeutic measures in stroke intervention $[37,38]$.

\section{Evolution of the Stroke Interventional Laboratories}

The field of stroke interventions has progressed dramatically in leaps and bounds in the last century, and is one of the most rapidly evolving disciplines in medicine. The first cerebral angiogram was performed in the 1930s [39, 40]. Fast forward 40 years of development and experience, femoral access for cerebral vasculature visualization was finally refined, and interventional techniques became widespread [41]. Exceedingly dependent on advancements in material science, computer engineering, and software development, the breadth of the catheter-based treatments of cerebrovascular disease has increased exponentially. This rapidly expanding specialty paralleled the increasing number of stroke interventional training programs with a wide variability in the training of the cerebrovascular specialist's background (neurosurgery, neurology, and radiology) [42, 43]. As the number of stroke interventional programs increased, the number of stroke interventional laboratories began to proliferate. 
With the advent of lumen-based cerebral angiography in conjunction with CT technology and ultrasound, stroke interventionalists increasingly began to identify and quantify the degree of cerebrovascular disease, and to explore the role of intervention techniques based on the imaging findings. Initially in 1970s, catheterization labs were few and limited to major academic centers. As training programs proliferated, by the 21st century, improved safety and simplicity of diagnostic procedures fostered the proliferation of laboratories in community hospitals with or without specialized neurological intensive care units, neurosurgical services, or even input from stroke specialists. According to data from the National Inpatient Sample in 2001, US hospitals are collectively treating $>300,000$ diagnostic and therapeutic angiographic procedures per year, including $>150,000$ carotid stenoses, and $>130,000$ cerebral angiographies, with special note that stroke interventional volumes are highly dependent on the success of recent endovascular stroke trials and technologies (including newer-generation stentriever and thromboaspiration devices), and expected sustainable growth into the next several decades [44].

Interventionalists have begun to increasingly utilize outpatient services and stroke interventional laboratories that are independently operating under the umbrella of comprehensive stroke centers with no specific uniform credentialing of the laboratory itself. In addition, EMS does not know whether appropriate time-sensitive stroke treatments will be provided to the stroke patients they bring to the nearest 'stroke center'. The terms 'primary stroke center', 'advanced stroke center', 'comprehensive stroke center', are loosely used, despite legislations in several states across the Unites States clearly mandating the appropriate use of the term for the appropriate level of stroke designation, increasing the level of confusion amongst EMS.

Clearly, there is a need to simplify stroke triage in the era of mechanical thrombectomy for LVO AIS into those facilities that can only provide 24/7 IV t-PA (i.e. drug delivery facility, stroke referral facility, or non-stroke intervention lab capable facility) versus those facilities that can also provide 24/7 stroke interventional treatments (i.e. drug plus device facility, stroke receiving facility, or stroke intervention lab capable facility). In addition, it is important to standardize the quality of stroke interventional laboratories worldwide, and there is an unmet need for what constitutes a high-quality stroke interventional laboratory, namely SILC criteria.

\section{The '7M Approach' to the Standardization of Stroke Interventional Laboratories (SILC Criteria)}

1 Manpower - personnel and supervision

2 Machines - physical facilities and angiographic equipment

3 Materials - medical device inventory, medications, and angiographic supplies

4 Methods - standardized protocols for stroke workflow optimization

5 Metrics (volume) - existing credentialing criteria for facilities and stroke interventionalists

6 Metrics (quality) - benchmarks for quality assurance

7 Metrics (safety) - radiation and procedural safety practices (table 1)

\section{Section 1: Manpower - Personnel and Supervision}

In order to sustain the daily operational components of a stroke interventional laboratory reliably and systematically, a certain critical mass of personnel is required. This section will outline all necessary physician and ancillary staff with experience in all aspects of delivering stroke interventional procedures and post-procedural care. 
Table 1. SILC recommended criteria for a successful stroke interventional laboratory

\begin{tabular}{ll}
\hline Manpower & $\begin{array}{l}\text { medical director, administrative director, stroke interventionalists, medical and } \\
\text { administrative staff, physician extenders, interventional fellows, interventional } \\
\text { technologists, interventional nurses, imaging technologists, stroke coordinator, } \\
\text { ancillary personnel, IRB and research coordinator, anesthesia support }\end{array}$ \\
\hline Machines & $\begin{array}{l}\text { physical angiography space, angiographic equipment, instrumentation and software, } \\
\text { biplane angiography with built-in CT capability, neuro-angio pack (prepackaged for } \\
\text { stroke intervention procedures), additional single plane or biplane angiography suite } \\
\text { for simultaneous stroke interventions or divert policy }\end{array}$ \\
\hline Materials & $\begin{array}{l}\text { medical device inventory with adequate par levels (minimum basic devices including } \\
\text { guide catheters, guidewires, balloon catheters, microcatheters, microguidewires, } \\
\text { embolic materials, and thrombectomy devices), equipment and instrumentation quality } \\
\text { control, medication storage system, crash cart }\end{array}$ \\
\hline Methods & $\begin{array}{l}\text { protocol development for the following: reporting study results as part of medical } \\
\text { record, listing procedure indications, informed consent, procedure preparation and } \\
\text { conduct, brain attack activation, management of contrast induced nephropathy, } \\
\text { management of radiation induced alopecia and dermatitis, special situations, i.e. } \\
\text { angiographic procedures for pregnant or pediatric patients }\end{array}$ \\
\hline Metrics: Safety & $\begin{array}{l}\text { Radiation safety, Contrast safety, air kerma and fluoroscopy time } \\
\text { credentialing and maintenance of procedure volume with respect to facility and stroke } \\
\text { interventionalists }\end{array}$ \\
\hline Metrics: Volume \\
\hline $\begin{array}{l}\text { quality assurance, patient outcomes, performance metrics, i.e. prehospital EMS } \\
\text { procedures }\end{array}$ \\
\hline
\end{tabular}

Standard 1.1 - Medical Director

Every stroke interventional laboratory must have one medical and one administrative (see section Standard 1.4) director. The medical director of the stroke interventional laboratory should be a physician with extensive experience and leadership qualities to maintain the optimal laboratory environment. The director should have an unrestricted medical license according to local state regulations, should be fellowship trained in neurointervention, and should be board certified in their respective primary disciplines, including neurology, neurosurgery, or radiology. The duties of the stroke interventional medical director are multifaceted and demand strong management skills. The medical director of the stroke interventional laboratory is necessarily involved in stroke system-wide national initiatives including active participation in 'door to needle' (IV t-PA) task forces for target stroke [18], 'door to perfusion' (stroke intervention) task force, telestroke (if it is the standard practice of the institution), nationwide systematic approach to stroke operations, and certification, review, and renewal process with certifying bodies such as AHA/ASA, TJC, DNV, or HFAP, and any current or future state regulatory bodies. The medical director must have performed over 100 procedures, including diagnostic cerebral angiograms and stroke interventional procedures performed over his or her career [45].

The medical and administrative directors are responsible for quality assurance, periodical review of staff performances, appropriate renewal of laboratory privileges, review performance of nonprofessional staff, and provide necessary training to personnel. The medical director is further responsible for quality control including morbidity and mortality. 
Shams et al.: Society of Vascular and Interventional Neurology (SVIN) Stroke Interventional Laboratory Consensus (SILC) Criteria

Standard 1.2 - Medical Staff Including Stroke Interventionalist

The physician in charge of the procedure, the attending physician, is the primary operator for the procedure. This person should be credentialed according to local and national standards, knowledgeable in all aspects of performing the procedure, including pre- and postprocedural management of the patient. Any assisting physicians or physician extenders, other than the attending, are considered to be secondary operators and must be documented and accounted in the informed consent. All procedures must be staffed with at least one primary operator and any number of secondary operators in order to safely and efficiently complete each procedure. All secondary operators must participate in quality assurance programs, including peer review. Physicians performing stroke interventions should be board eligible or board certified.

Standard 1.3 - Physician Extenders and Interventional Fellows

Trainees or physicians in fellowship are considered secondary assisting operators and must be directly supervised by the primary operator. Physician extenders, including physician's assistants and nurse practitioners, registered nurses, or technologists, may not be primary operators, and all clinical decision making must reside with credentialed physician operators.

Physician extenders should demonstrate proficiency in the technical and cognitive aspects of stroke intervention, and the current standards of care in stroke guidelines, stroke operations, and existing nationwide initiatives for efficiency and stroke outcome improvements. The extenders must be knowledgeable in preprocedural evaluation, indications, pathophysiology, current acute ischemic and hemorrhagic stroke treatments, and radiation safety guidelines. All extenders must be familiar in application of diagnostic catheterization data regarding the procedure and standards established by the AHA/ASA, American Neurological Association (ANA), and SVIN. Nurses, physician's assistants, and technologists may be able to assist in place of trainees but require greater supervision during all aspects of the procedure. Specialized training in stroke interventional care and procedures are required, with documented case logs, subject to review by medical and administrative directors.

The primary goal of the stroke interventional fellow is to learn current stroke interventional procedures and diagnostic cerebral angiograms. In 2000, Accreditation Council for Graduate Medical Education (ACGME) approved guidelines delineating training qualifications, and in 2003, ACGME broadened the scope of the cerebrovascular expert training from neurology, neurosurgery, or neuroradiology backgrounds. Fellows or trainees, despite background discipline, will be trained in the metrics of procedural performance, pre- and postprocedural management, and therefore acquire the skills to become qualified attending physicians. Trainees may perform all functions of the procedure as the primary operator, but only under the direct supervision of a credentialed physician who assumes responsibility for the procedural results. All trainees must comply with ACGME and existing credentialing guidelines required to perform various angiographic procedures of different complexities, and eventually reach the status of a primary operator [43, 45-47] (table 2).

Standard 1.4 - Technical/Administrative Director

The technical/administrative director is responsible in conjunction with the medical director for the quality control of the stroke interventional laboratory. The administrative director should oversee control over patient scheduling, equipment maintenance, budget preparation, monitoring radiation doses, organization of conferences for laboratory personnel, and regular reports on laboratory activity. The administrative/technical director must work in close cooperation with the medical director to establish basic standards for personnel, maintenance, quality assurance, quality improvement, continuing education, and any financial decisions that directly impact the operations of the stroke intervention laboratory. 
Shams et al.: Society of Vascular and Interventional Neurology (SVIN) Stroke

Interventional Laboratory Consensus (SILC) Criteria

Table 2. Existing and SILC-recommended credentialing standards for stroke interventionalists

\begin{tabular}{|c|c|c|c|c|}
\hline Procedure & Existing guidelines for initial credentialing & $\begin{array}{l}\text { Existing guidelines } \\
\text { for maintenance }\end{array}$ & Special notes & $\begin{array}{l}\text { SILC-recommended volume of } \\
\text { procedure for initial } \\
\text { credentialing and maintenance } \\
\text { of proficiency }\end{array}$ \\
\hline $\begin{array}{l}\text { Diagnostic } \\
\text { angiography }\end{array}$ & $\frac{\text { ACGME [80] - } 50}{\text { Neurovascular coalition [33] - } 100}$ & $\begin{array}{l}\text { SCAI/SVMB/SVS } \\
\text { consensus } \\
\text { statement [81] - } 50\end{array}$ & & $\frac{100 \text { for initial credentialing [45] }}{50 \text { annually for maintenance }}$ \\
\hline $\begin{array}{l}\text { Intra-arterial } \\
\text { thrombolysis and } \\
\text { mechanical } \\
\text { thrombectomy }\end{array}$ & $\begin{array}{l}\text { ACGME - no existing guidelines set forth } \\
\text { specifically for thrombolysis procedures } \\
\text { IMS study protocol [82] - board eligible or } \\
\text { certified physician with } 100 \text { diagnostic } \\
\text { cerebral angiographies as primary } \\
\text { operator PLUS a minimum } 1 \text { year } \\
\text { fellowship in endovascular neuroradiology } \\
\text { meeting requirements of ACGME that } \\
\text { includes } 10 \text { intra-arterial thrombolysis } \\
\text { procedures }\end{array}$ & None & $\begin{array}{l}\text { IMS protocol in absence of formal } \\
\text { training: completion of residency } \\
\text { and } 1 \text { year fellowship in accredited } \\
\text { program or equivalent, with } \\
\text { training in cerebral angiography at } \\
\text { an approved program with } 200 \\
\text { cerebral angiograms, } 50 \\
\text { interventional neurology } \\
\text { procedures under a director with } \\
\text { adequate experience and at least } \\
10 \text { intra-arterial thrombolysis } \\
\text { procedures }\end{array}$ & $\begin{array}{l}100 \text { diagnostic cerebral } \\
\text { angiographies PLUS } 30 \text { stroke } \\
\text { interventional procedures } \\
\text { (including use of } \\
\text { microcatheters) PLUS } 10 \text { intra- } \\
\text { arterial stroke thrombolysis/ } \\
\text { mechanical thrombectomy } \\
\text { procedures [44] }\end{array}$ \\
\hline $\begin{array}{l}\text { Carotid angioplasty } \\
\text { and stent placement }\end{array}$ & $\begin{array}{l}\text { ACCF/SCAI/SVMB/SIR/ASITN } 2007 \\
\text { consensus document [70] - } 100 \text { cerebral } \\
\text { angiograms and EITHER } \\
25 \text { noncarotid stents with } 4 \text { supervised } \\
\text { carotid stent procedures with } 16 \text { h of CME, } \\
\text { OR } 10 \text { supervised carotid angioplasty and } \\
\text { stent placement procedures with } \\
\text { acceptable results } \\
\text { SCAI/SVMB/SVS Consensus document } \\
\text { [81] - } 30 \text { cervicocerebral angiograms, } \\
\text { with } 15 \text { as primary operator, and a } \\
\text { minimum of } 25 \text { supervised carotid } \\
\text { interventions, with } 13 \text { as primary operator }\end{array}$ & $\begin{array}{l}\text { Varies depending } \\
\text { on institutional, } \\
\text { state, and federal } \\
\text { guidelines }\end{array}$ & & $\begin{array}{l}200 \text { diagnostic cerebral } \\
\text { angiographies PLUS } 10 \text { carotid } \\
\text { angioplasty and stenting } \\
\text { procedures for initial } \\
\text { credentialing [48] }\end{array}$ \\
\hline
\end{tabular}

Standard 1.5 - Interventional Technologist(s)

A variety of knowledgeable technical personnel may be required for the sake of efficiency in operating a stroke interventional laboratory, and one person is unlikely to possess all different expertise. At least one technologist, preferably a certified radiological technologist, should be skilled in radiographic and angiographic imaging principles and techniques. This technologist should be experienced in the proper performance of X-ray generators, cine pulse systems, image intensification, automatic film processing, pressure injection systems, video systems, and cine cameras. He or she in conjunction with radiological service engineers should be responsible for routine care and maintenance of radiological equipment. The basic ability to troubleshoot equipment in the stroke interventional laboratory is advantageous. This technologist, in cooperation with radiation physicist, must monitor radiation safety techniques for both the patient and laboratory personnel. Immediate availability of radiological engineer in the event of the equipment failure is highly desirable.

\section{Standard 1.6 - Interventional Nurse(s)}

The utility of nursing personnel may vary in type and number in the stroke interventional laboratory, depending on caseload and mix, and may include nurse practitioners, registered nurses, licensed vocational or practical nurses, or nursing assistants. In most laboratories, the nursing supervisor is a registered nurse. This nurse must be familiar with all overall function of the laboratory, help set the tone of patient surroundings, and influence the efficiency and safety of procedures. Nursing staff may directly participate in observation, emergency response, and charge the pre and post-procedural holding areas. Nursing staff must assist with hemodynamic and neurological monitoring for regular documentation of vital signs and neurological assessments. 
Shams et al.: Society of Vascular and Interventional Neurology (SVIN) Stroke Interventional Laboratory Consensus (SILC) Criteria

Standard 1.7 - Imaging Technologist(s) for CT/MRI

Although imaging technologists are not necessarily required to be present during the procedure in the stroke interventional laboratory, if advanced imaging services such as MRI or CT are required, such services should be readily accessible. The interventional technologist staffing levels should be sufficient to provide a technologist per procedure room. Any imaging technologist involved in patient management should be familiar with stroke interventional techniques, imaging protocols per facility, and national guidelines related to stroke, and must also participate at regular intervals in continuing education, quality assurance, and quality improvement.

Standard 1.8 - Neurological Assessment Examiner

Neurological assessments must be performed before, during, and after any stroke intervention including diagnostics at regular intervals, as appropriate.

Standard 1.9 - Stroke Coordinator and Ancillary Personnel

Stroke coordinators are the cornerstones of any stroke program, and responsible for multiple tasks including quality assessments, get-with-the-guidelines compliance, monitoring IV t-PA administration, and monitoring time-based metrics in stroke care for quality improvement. The stroke coordinator is equally essential to the stroke interventional team, and must actively participate in numerous task forces of the stroke interventional lab such as 'door to needle' task force, 'door to perfusion' task force, quality assurance, and quality improvement. Stroke coordinators and all other ancillary personnel must be familiar with national guidelines in stroke interventional care. They are strongly recommended to participate in national conferences for continuing education.

Standard 1.10 - Institutional Review Board and Research Coordinator

SVIN strongly recommends any stroke interventional laboratory to participate in national registries, data reporting, and statistical analysis of information. Though historically most stroke interventional laboratories have been associated with academic facilities that reflexively participate in large trials, the stroke interventional laboratory, despite its setting, academic or community is a fertile ground for clinical trials and advanced research. Institutional review board (IRB) and research coordinators should be utilized and strongly recommended for both clinical research and patient registries. IRB and research coordinators may be from various different disciplines including, trial design, nursing, and/or work experience. It is essential the IRB and research coordinator is knowledgeable in stroke interventional clinical management and literature. They are strongly recommended to participate in national conferences for continuing education.

Standard 1.11 - Anesthesia Support

The utility of general anesthesia during stroke interventional procedures has not been validated and remains a topic of controversy [48]. The majority of stroke interventionalists nationally practice conscious sedation or monitored anesthesia for LVO AIS cases unless the patient needs airway protection, in which case general anesthesia is provided [49]. In a fully credentialed stroke interventional laboratory, full anesthesia support must be available for complicated cases during which intubation and sedation is indicated. It is not recommended to practice routine general anesthesia for all patients and must be reviewed by individual cases [50]. Level 1 conscious sedation practices are recommended for stroke interventional procedures, under the supervision of appropriately trained providers. 
Shams et al.: Society of Vascular and Interventional Neurology (SVIN) Stroke Interventional Laboratory Consensus (SILC) Criteria

\section{Section 2: Machines - Physical Facilities and Angiographic Equipment}

Standard 2.1 - Physical Space

Basic equipment and laboratory need to be located in a setting that provides electrical service, air conditioning, air exchange, sterile conditions, room and task lighting, telephone, computer, and patient amenities required for these types of procedures. Whether the stroke interventional laboratory is part of a hospital or outpatient facility, it must be of sufficient size to accommodate imaging and nonradiographic equipment, provide easy access to the patient from multiple approaches and necessary life support equipment. Stroke interventional laboratory is ideally situated close to other modalities of imaging including CT, MR, as well as proximity to the emergency room for the purpose of minimizing time to treatments in acute stroke.

The interventional laboratory should also factor in accessory components such as staff changing areas, tele-stroke station, dictation station, computers for ease of access to medical records, conference areas, patient holding rooms, and family counseling areas. While these elements are not necessarily directly part of the laboratory space, they should be close and preferably adjacent to the interventional laboratory.

Standard 2.2 - Angiographic Equipment, Instrumentation, Software

In addition to the cognitive knowledge of stroke interventional techniques, radiographic equipment is also rapidly evolving after years of little change in interventional neuroradiology. Fixed-installation fluoroscopy equipment, designed and specified for stroke interventional procedures, is preferable $[51,52]$. Considerations in equipment parameters include but are not limited to appropriately sized image receptor, permanent recording modules such as digital subtraction versus cine, fluoroscopy tube focal spots, output, and heat load with cooling capacity, generator capacity, software modules, local storage capacity for images, DICOM ability to integrate with PACS, dose management tools custom to procedure performed, and limitations of patient weight on angiography table.

Biplane angiography with DSA, high-resolution image intensifier, and imaging chain, digital subtraction imaging $(1,024 \times 1,024$ matrix is ideal $)$ should be standard [53]. Road map fluoroscopy capabilities with and without simultaneous live unsubtracted imaging are also required. A technologist must document fluoroscopy time, and dosimetry should be used.

Biplane and 3-D angiography are essential, and angiography equipment with in-built CT capability tools for stroke interventional procedures are strongly recommended. For procedures specific to stroke intervention, biplane angiographic equipment, despite high costs, has marked advantage to single-plane angiography [54]. Single plane with high specifications may be utilized in diagnostic angiograms, but biplane is preferable. Cone-beam CT capability may be useful for stroke interventional procedures [55]. Depending on patient size and user preference, large bore CT scanners with CT fluoroscopy may be utilized for CT-guided interventions. As there are times when there can be 2 patients with an LVO AIS presenting to the facility, a second bi-plane or single-plane angiography suite should be available as a back-up for emergent simultaneous stroke interventional treatments and neuroangiography. In addition, stroke interventional laboratory should have a written protocol on how they would manage two simultaneous LVO AIS patients, along with necessary transfer protocols or divert notification policies to EMS if appropriate.

Stroke-specific angiography packs should include necessary equipment and materials and play a key role in minimizing errors in setup and increasing time efficiency. Materials such as sterile scalpels, needles of various gauges, gauze, femoral drapes, towels, stopcock, gallipots, and fluoroscopy cover, color-coded labels, neuroangiography drape and pouch, waste bag, trolley cover, and needle containment device are examples of proposed contents of a stroke intervention angiography pack (table 3). 


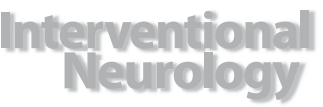

Table 3. Neuro-angio pack (courtesy of Texas Stroke Institute)
Intervent Neurol 2016;5:1-28

(C) 2016 S. Karger AG, Basel www.karger.com/ine

Shams et al.: Society of Vascular and Interventional Neurology (SVIN) Stroke Interventional Laboratory Consensus (SILC) Criteria

\begin{tabular}{|c|c|}
\hline Required materials & Additional \\
\hline Basin ring & Torque device \\
\hline 2 bowls for guidewires with tabs & $150-\mathrm{cm}$ (diagnostic) or $180-\mathrm{cm}$ \\
\hline Chlorhexidine & (intervention/exchange) glidewire \\
\hline Cup & Fluid management pack \\
\hline Table cover $50 / 90$ & 3 pressure bag drip lines \\
\hline Femoral drape & 5-Fr sheath \\
\hline Fluoroscopy drape & Micropuncture kit \\
\hline \multicolumn{2}{|l|}{ Fluoroscope sterile cover } \\
\hline \multicolumn{2}{|l|}{ Foam for sharps } \\
\hline \multicolumn{2}{|l|}{ Gauze $4 \times 4$} \\
\hline \multicolumn{2}{|l|}{ Sterile gowns $\times 2$} \\
\hline \multicolumn{2}{|l|}{ Intake glass } \\
\hline \multicolumn{2}{|l|}{ Label sheets } \\
\hline \multicolumn{2}{|l|}{ Large prep tray } \\
\hline \multicolumn{2}{|l|}{ Utility marker } \\
\hline \multicolumn{2}{|l|}{ Microscope tape } \\
\hline \multicolumn{2}{|l|}{ Mosquito forceps $\times 1$} \\
\hline \multicolumn{2}{|l|}{ 18-gauge needle $\times 1$} \\
\hline \multicolumn{2}{|l|}{ 22-gauge needle $\times 1$} \\
\hline \multicolumn{2}{|l|}{25 -gauge needle $\times 1$} \\
\hline \multicolumn{2}{|l|}{ Nonadherent pads to wipe wires } \\
\hline \multicolumn{2}{|l|}{ Sterile towels ×8 (1 pack) } \\
\hline \multicolumn{2}{|l|}{1 OR sterile scissors } \\
\hline \multicolumn{2}{|l|}{ Waste bag } \\
\hline \multicolumn{2}{|l|}{ Stopcock 3-way ×2 } \\
\hline \multicolumn{2}{|l|}{ Stopcock low pressure 1 way $\times 2$} \\
\hline \multicolumn{2}{|l|}{ Syringe $10 \mathrm{ml} \times 5$} \\
\hline \multicolumn{2}{|l|}{ Syringe $20 \mathrm{ml} \times 1$} \\
\hline \multicolumn{2}{|l|}{ Syringe $5 \mathrm{ml} \times 4$} \\
\hline \multicolumn{2}{|l|}{ Yellow plunger $3 \mathrm{ml} \times 1$} \\
\hline \multicolumn{2}{|l|}{ Syringe $3 \mathrm{ml} \times 4$} \\
\hline \multicolumn{2}{|l|}{ Table cover } \\
\hline \multicolumn{2}{|l|}{ Table $18 \times 26$} \\
\hline Tray foam & \\
\hline
\end{tabular}

Stroke interventional laboratories should have a fully stocked code cart located in a visible and easily accessible area within the angiography suite. Necessary and recommended equipment also includes items needed for anesthesia. There should be $24 / 7$ access to advanced imaging techniques including MRI, CT, MR perfusion, CT perfusion, and CT angiography as all these modalities aid in diagnosis and management.

Section 3: Materials - Medical Device Inventory, Medications, and Angiographic Supplies

Standard 3.1 - Medical Device Inventory

A fully certified stroke interventional laboratory should achieve a certain degree of preparedness to handle any possible stroke interventional cases that may present itself at any time. While it is impossible to predict specific catheters, guidewires, embolic particles, or thrombectomy devices required for each procedure, multiple sizes and types of each basic device should be stocked at all times with adequate par levels for each device. Basic devices include guide catheters, guidewires, balloon catheters, microcatheters, microwires, embolic 
Shams et al.: Society of Vascular and Interventional Neurology (SVIN) Stroke Interventional Laboratory Consensus (SILC) Criteria

materials, and thrombectomy devices. In addition, there should be an inventory tracking system to assess the par levels of various devices on an ongoing basis.

Standard 3.2 - Equipment and Instrumentation Quality Control

The modern and fully equipped stroke interventional laboratories will likely contain many sophisticated radiographic, electronic, and computer-based systems, which require maintenance and troubleshooting. The biplane and X-ray imaging system should be especially monitored for early deterioration. 24/7 access to IT help desk, backup systems for storage of essential information pertaining to any stroke interventional and diagnostic procedures, and low cost storage media are crucial. Adequate digital storage and capabilities for archiving of images are crucial so that there is no risk of accidental deletion of images acquired during a stroke interventional procedure due to lack of file storage or digital memory space.

Standard 3.3 - Pharmacy and Medications

The stroke interventional laboratory, in addition to hemodynamic monitoring capabilities, must also be equipped to treat certain medical and neurological emergencies such as hypertension, raised intracranial pressure, acute thrombosis, acute life-threatening bleeding, and seizures, to name a few. For this reason, the stroke interventional laboratory should contain a medication storage system (for example Pyxis, Omnicell) as per institution preference. If the medication storage system is not directly in the stroke interventional lab, it must be in close proximity to one, where nursing staff can readily access emergency medications. Medications should be administered by nursing staff and documented appropriately similar to any other setting where medications are administered. Speedy access to the facility's pharmacy for medications that are not stocked in the storage system is essential for patient safety.

Section 4: Methods - Standardized Protocols for Stroke Workflow Optimization

Standard 4.1 - Reporting of Study Results in Medical Record

The formal procedural report after a stroke interventional procedure should contain some critical information. Operators should clearly state indications, time course of procedural events, and all times and doses of medications administered. All documentation must be maintained as part of permanent medical records. The use of all catheters, sheaths, and special guidewires should be documented. Vital signs and neuro checks must be monitored at regular intervals and also maintained as part of the medical record.

The format of the stroke intervention procedure report should include key elements including name of procedure, indication, primary and all secondary operators, a brief description of pertinent history and clinical events, anesthesiologist (if present), duration of procedure, access technique with findings, description of named procedure itself with pertinent positive and negative findings, vascular closure device use and technique, and documentation of family discussion explaining key findings of stroke interventional procedure. Documentation of procedural and hemodynamic records should be maintained at least 7 years or longer as per institution and state standards. The procedural reports should contain appropriate scales and scores that assess the severity of the disease (e.g., National Institute of Health Stroke Score, modified Rankin score, etc.). In addition, the procedural reports should also contain the appropriate time-based metrics essential in the management of LVO AIS (e.g., time from last known normal, door to groin times, groin to perfusion times, etc.).

The basic cerebral angiography procedure report should begin with the indication for the procedure followed with description of the initial vascular access and progress to describing each DSA runs of the aortic arch and its branches. Detailed description of the procedure should include all devices used, all pertinent positive and negative findings. 
Shams et al.: Society of Vascular and Interventional Neurology (SVIN) Stroke Interventional Laboratory Consensus (SILC) Criteria

Standard 4.2 - Procedure Indications and Informed Consent

Procedural information should be presented objectively, honestly, and outcomes data should be systematic and standardized. Prior to all procedures, both diagnostic and intervention, standard informed consent and time-outs should be obtained prior to procedure. Informed consent should note all participants of the procedure and describe all possible procedures including ad-hoc interventions, should they become a consideration. Clinical research studies require special attention, with patient safety prioritizing over all aspects of investigational protocol.

Standard 4.3 - Procedure Preparation and Conduct

Although no rigid protocol is applicable to all laboratories, certain procedural issues, such as premedication, should be addressed. In cases where conscious sedation is required, fixed protocols should be maintained during the procedure. Sterile preparation is mandatory for all vascular access sites. All operators must wear masks, caps, and eye protection to prevent accidental operator contamination.

Proper procedural technique includes adequate injections of cerebral vasculature including the aortic arch, and use of multiple views with appropriate radiographic angulation for visualization of the cerebral vascular structures. Patients should have adequate monitoring including hemodynamic monitoring of basic vital signs (blood pressure, heart, rate, respiratory rate, and oxygen saturation) along with frequent neurological checks, which must be documented in flow sheets during the procedure.

Post-procedural hemostasis may be achieved by several means including manual pressure, mechanical compression devices, or percutaneous closure devices. Patients should be monitored after procedure for hematoma and pseudoaneurysms, and event rates of each method and each device must be tracked.

Antiplatelet agents, including aspirin and Plavix, do not need to be withheld before cerebral angiography. Warfarin should be discontinued until INR is $<1.8$, if clinically indicated after a discussion of the risks and benefits with the patient or family. If necessary, warfarin may be reversed with FFP or vitamin $\mathrm{K}$ as per pharmacy protocol.

Standard 4.4 - Brain Attack Activation Protocol for Nurses, Imaging Technologists, Anesthesiologists, ED Team, and Stroke Interventionalists

The stroke interventionalists must operate within a structured framework of support staff and services from the EMS, ED, radiology, and stroke interventional laboratory. AIS patients, from the time of first contact in the field by EMS, need to be triaged according to treatment modality, either IV t-PA, stroke intervention, or medical management focused on secondary prevention. In efforts to improve outcomes and reduce mortality in AIS patients, national guidelines, protocols, and algorithms have been developed and organized into a systems operation for facilitating the selection of patients eligible for IV t-PA administration. Fifty-three international trials in the past several decades, including the NINDS and ECASS trials [56, 57], demonstrate a significant reduction in 3-month mortality and morbidity for stroke patients who undergo recanalization.

Identifying and establishing criteria for selecting patients ideal for stroke intervention has historically been less straightforward, based on results from trials such as IMS-III and MR-RESCUE [58, 59]. Currently, between the results of the FIRST study [60], which demonstrates the natural history of stroke to be devastating with nearly $80 \%$ of LVO AIS patients either dead or disabled for life if left untreated, and a clear benefit of endovascular revascularization therapies for LVO AIS in several recent landmark trials including the MR-CLEAN trial $[2,19]$, we have reached a paradigm shift, where there is an imminent unmet need to create advanced algorithms that facilitate the identification of LVO AIS patients appro- 


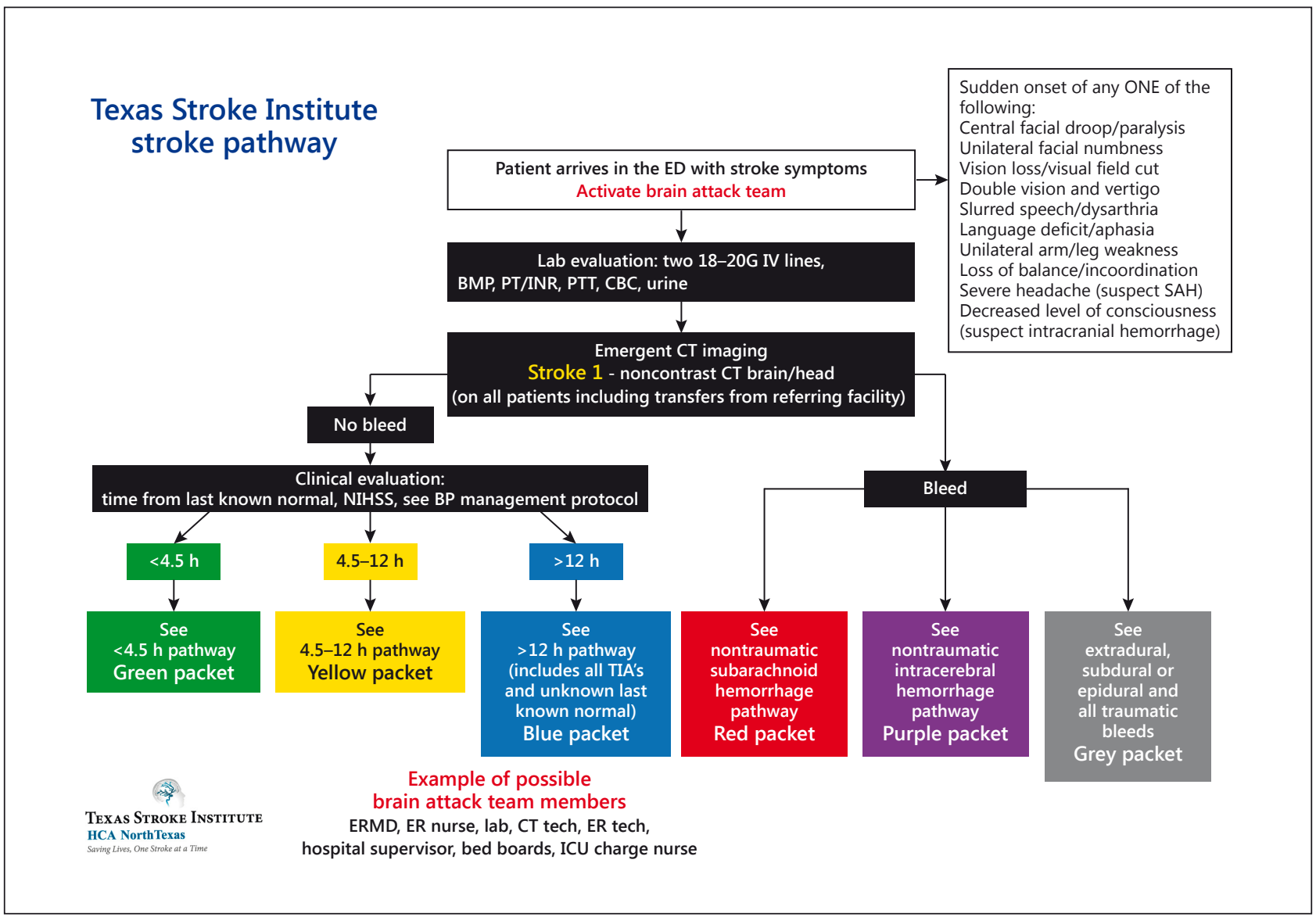

Fig. 5. ED-based rapid brain attack triage algorithm for identification of patients eligible for stroke intervention. Algorithm courtesy of Texas Stroke Institute.

priate for stroke interventional procedures from the moment of initial contact with EMS [60].

The SVIN Writing Committee endorses that in compliance with brain attack coalition guidelines for acute stroke-ready, primary, and comprehensive hospitals, when taking care of stroke patients (ischemic and hemorrhagic), every stroke system of care must have clearly defined protocols in place to facilitate initial triage in the field, timely and resource-efficient management in ED, 'Get with the Guidelines' compliance during inpatient admission, and discharge to rehabilitation for those eligible, in efforts to improve outcomes of stroke patients as part of a national initiative. In addition, the writing committee further endorses the establishment of clear algorithms and care paths for identifying and managing stroke patients eligible for interventions via catheter-based treatment modalities (fig. 5) [61]. All medical staff, including physicians, nursing, and ancillary staff, must be educated in such protocols, and a system must be established where alert system may be activated easily and rapidly.

Standard 4.5 - Protocol for Management of Contrast-Induced Nephropathy

Patient's with contrast allergies should receive nonionic contrast and premedicated with steroids or antihistamines, as per pharmacy protocol of the institution. Patients with renal insufficiency should be hydrated before and after procedure, and acetylcysteine should be considered in necessary circumstances. 
Fasting patients with diabetes mellitus should receive a reduced dose of insulin in the morning of the procedure. Diabetes patients treated with metformin who have mild renal insufficiency rarely have been reported to develop profound lactic acidosis after receiving radiographic contrast. Metformin dose should be held on the day of the procedure and not started until creatinine stabilizes usually $48 \mathrm{~h}$ after the procedure.

Standard 4.6 - Protocol for Management of Radiation-Induced Alopecia and Dermatitis

Radiotherapy can result in certain inevitable side effects, radiation-induced cutaneous side effects that include acute and chronic dermatitis, alopecia, and systemic side effects. Damage may occur at gross and molecular levels. Changes usually occur 2-5 weeks after exposure, are usually self-limiting and tend to be largely temporary, and transiently resolve with good prognosis after 6 months $[62,63]$. These adverse effects are a direct result of radiation dose accumulation, and certain precautions should be kept in mind. Stroke interventionists should be aware of these effects and strict adherence to maximum radiation dose policies should be maintained.

Standard 4.7 - Special Considerations: Pediatric and Pregnancy

Conditions such as the pediatric patient or pregnancy require special considerations. The pediatric population is particularly vulnerable to certain complicated cerebrovascular diseases such as sickle cell disease, arteriovenous malformations, moyamoya, and vasculitis, to name a few [64]. Endovascular procedures are viable options for diagnostics and therapeutics. Goals of care are to define intra- and extra-cranial vasculature and hemodynamics, as well as provide therapeutic benefits. Stroke interventionalists are highly specialized and exist in scarce ratios to serve patient populations, and pediatric expertise and volume are even scarcer. Children under the age of 18 years of age must undergo informed consent from parent or guardian. Patients appropriate for outpatient scheduled procedures must be selected carefully, and overnight admission for monitoring should be considered based on the condition of the patient. Early discharge for both pediatric and pregnant patients must factor in age and weight, parent or patient reliability, travel time and distance, duration of procedure, time of completion, hemodynamic stability, and estimated blood loss. All procedures on pregnant patients must be performed with full informed consent of the patient as well as in conjunction with obstetricians to help guide the decision-making process.

\section{Section 5: Metrics (Volume) - Existing Credentialing Criteria for Facilities and Stroke Interventionalists}

Standard 5.1 - Facility Volume (table 2)

The stroke interventionalists must fulfill and maintain basic requirements in cognitive knowledge, technical expertise, and clinical competency throughout the length of the stroke interventionalist's career to ensure safe and efficient delivery of quality care in potentially life-threatening, critical cerebrovascular diseases [31, 32, 42, 65, 66]. Further, the number of stroke interventional laboratories within primary and comprehensive stroke centers are rapidly increasing [67] without significant oversight. Although the use of specific minimum number of cases to define the quality of operator performance is an imperfect solution to oversee provider and laboratory quality, potential laboratories performing stroke interventional procedures without appropriate oversight and established quality assurance systems pose significant threats to quality of care and patient outcomes. Further, according to multisociety statement for quality assurance in stroke interventional procedures, increased operator experience is inversely proportional to adverse event rates [31, 32, 47]. The SVIN SILC Writing Committee endorses the tracking and reporting of procedural volumes by 
operator and laboratory to ensure safety and quality $[7,68]$. Despite the background discipline of the stroke interventionalist, whether neurology, neurosurgery, or neuroradiology, competency and credentialing must be tracked in a standardized uniform manner for each laboratory in order to equally assess the procedural skills and adequacy of every stroke interventionalist.

The diagnostic cerebral angiogram is the cornerstone and foundation for all modalities of interventional stroke therapies, including carotid artery angioplasty and stenting of atherosclerotic vessels, interventional angioplasty and stenting, coiling and embolization of cerebral aneurysms, embolization of epistaxis and vascular malformation procedures. The writing committee has accounted for the existing recommendations of several overseeing organizations including ACGME, procedure-specific credentialing through hospital facilities, TJC, DNV, HFAP, Center for Medicare and Medicaid Services (CMS), the Neurovascular Coalition, and the Brain Attack Coalition. All recommendations in this current publication comply with existing recommendations and further standardize them for uniform tracking and reporting.

Diagnostic Cerebral Angiograms. Existing regulations from various committees recommend that each trainee or fellow participates in a minimum number of procedures for initial credentialing throughout the training period ( 50 cerebral angiograms per ACGME guidelines and 100 per multi-society statements from the American Academy of Neurology (AAN), American Society of Interventional and Therapeutic Neuroradiology (ASITN), joint section for cerebrovascular neurosurgery, Congress of Neurological Surgeons (CNS), American Society of Neuroradiology (ASNR), and Ad Hoc Writing Group [31]. Experienced operators should oversee the trainees in diagnostic cerebral angiograms with adequate documentation of formal training maintained as part of records. Trainees should have adequate experience as primary and secondary operator, always under supervision. The number of procedures, the success, failure, and complication rates, and outcomes should be documented. In compliance with the above multi-society guidelines, trainees must complete at least 100 cerebral diagnostic angiograms for initial credentialing, and 50 cerebral diagnostic angiograms annually for maintenance of operator skills.

Intra-Arterial Thrombolysis and Mechanical Thrombectomy. Advanced stroke treatment delivery beyond IV t-PA, i.e. stroke interventions including intra-arterial thrombolysis and mechanical thrombectomies, should be performed in a qualified stroke interventional laboratory to maintain the highest quality standards. ACGME requires at least 10 intra-arterial thrombolytic procedures as part of the accredited ACGME endovascular surgical neuroradiology fellowship. Due to the scarcity of ACGME-accredited programs (requires only 1 year of neurointerventional training), most stroke interventionalists will choose to train in nonACGME programs (duration of 2 years and beyond for better exposure and experience), the SVIN Writing Committee feels that compliance with recent guidelines as well as ACGMErecommended 10 intra-arterial thrombolytic cases are adequate for sufficient experience for stroke intervention [43]. When performing such procedures, stroke interventionalists must take into special consideration the careful selection of patients eligible for stroke intervention and potential intracerebral hemorrhage resulting in increased mortality and morbidity.

Carotid Angioplasty and Stent Placement. Similar to the 2007 Clinical Expert Consensus on Carotid stenting statement from ACCF/SCAI/SVMB/SIR/ASTN [69], which accounts for the previously published guidelines from ASITN/ASNR/SIR in 2003 [47] and AAN/AANS/ ASITN/ASNR/CNS/SIR in 2005 [70], the SVIN Writing Committee recommends that every stroke interventionalist perform at least 25 carotid angioplasty and stent procedures during fellowship under supervision for adequate competency. Based on operator competencies and complication rates of the SAPPHIRE, CREST, EVA-3S, and CAPTURE trials, the threshold of 25 carotid angioplasty and stent procedures appears to yield a satisfactory skill sets. The utility of industry-sponsored courses is unclear, and their role in improving operator technical skills 
has never been validated. Further, an individual stroke interventionalist must comply with regulations from ACGME, institutional, state, and federal guidelines to become fully credentialed and maintain an adequate volume per year per operator to remain fully proficient in carotid angioplasties and stent placement.

Intracranial Stent Placement. Atherosclerotic intracranial stenosis is responsible for approximately $10 \%$ of all strokes every year. Though not all intracranial stenosis patients are eligible for stroke intervention, appropriately selected patients with significant risk of recurrent strokes may be eligible for stroke intervention with intracranial stents [71]. AHA/ ASA and TJC do not require minimum volume of intracranial stents as part of the comprehensive stroke center certification. There are no available data for assessing training requirements for intracranial stents. The SVIN SILC Writing Committee recommends that stroke interventional laboratories document and participate in registries for intracranial stenting, so future data and outcomes may be derived.

\section{Section 6: Metrics (Quality) - Benchmarks for Quality Assurance}

Standard 6.1 - Quality Assurance

Quality assurance begins with clinical proficiency among the operators in the stroke interventional laboratory. The challenges in assessing operator efficiency are multifold, and quality metrics regarding cognitive knowledge, procedural skill, clinical judgment, and procedural outcomes are all equally important factors. In order to ensure quality assurance of the performance of the laboratory unit as a whole, a continuous quality improvement program should be incorporated into the overall design of the stroke interventional laboratory, with key documentation and regular tracking of quality metrics. These measures are to be overseen by the medical and administrative directors.

Standard 6.2 - Patient: Outcomes

Outcomes related to complications for diagnostic cerebral angiograms should be very low [65]. Diagnostic accuracy and adequacy are obviously important parameters but rarely tracked. Major complications, including death, intracerebral hemorrhage, or particulate embolization from procedures should be monitored and evaluated against the severity of the patient population (referral bias) as well as benchmarks from landmark trials $[2,65]$.

The minimum number of studies required confirming adequate skills in neurological diagnostic catheterization procedures have never been validated. According to practice guidelines from American Society of Neuroimaging (ASN) and SVIN [45], 100 diagnostic cases per operator, 50 recommended/year is adequate for proficiency in skills. Though risks of diagnostic cerebral angiograms are low, quality improvement measures should be operative and hold precedence. A low-volume operator, $<50$ numbers/year, should only work in highvolume laboratory $>100$ numbers/year, and do so with adequate mentoring.

Equipment requirements and management are detailed in a section above (see section 2.2). Each aspect of the radiographic equipment system should meet standard performance expectations for the safe and quality delivery of patient care.

Standard 6.3 - Performance Metrics: Prehospital EMS Activation and Transport

According to the AHA/ASA and the Brain Attack Coalition, standard metrics for measuring quality of care at comprehensive stroke centers have been recommended as part of the national initiative to provide stroke care within service-based levels of stroke centers, and thus improve outcomes in an otherwise debilitating disease [72]. Care paths within systems of stroke care must be organized from the moment of initial contact and triage and follow all the way through discharge disposition. Segments of operation should be divided into prehospital (EMS) metrics, hospital (ED) metrics, and laboratory (stroke interventional laboratory) 
metrics. From the time of initial contact (first medical contact) by EMS operators in the field, performance metrics must be tracked, which helps further identify areas of missed opportunities in improving outcomes in stroke patients. Though national EMS performance metrics in stroke systems, or other similarly organized levels of care such as trauma, have not been clearly established in medical literature, the chain effect of efficient operations at the EMS level trickling down to efficient ED response, admission, and discharge is indirectly implied.

Standard 6.4 - Performance Metrics: In-Hospital ED Triage

Upon arrival of the stroke patient at the appropriate ED, clear concise protocols must be in place for identification of subtype of stroke and rapid delivery of appropriate stroke intervention treatments. Performance metrics measurement at the ED level ensures that stroke hospitals are able to reach stroke onset to IV t-PA, 'door to needle', 'door to perfusion', and other efficiency benchmarks, and further identify areas of improvements in efficiency. The SVIN SILC Writing Committee recommends that all Stroke Interventional Laboratories track key performance metrics in ED and involve the leadership of ED staff in institutional task forces.

Standard 6.5 - Performance Metrics: Diagnostic Procedures

Radiology metrics, such as 'door to CT', 'door to CT angiography', 'door to MRI', are also crucial in improving stroke care delivery. All stroke interventional laboratories must note and document time to imaging, to identify potential improvements or changes that may hinder delivery of stroke interventions to eligible patients. Stroke hospitals must develop taskforces and radiology initiatives for tracking and optimal stroke care delivery.

Standard 6.6 - Performance Metrics: Stroke Intervention Procedures

A means to ensure continuing education and most current cognitive knowledge of all staff involved in the stroke interventional laboratory should be established and adhered to. Key metrics reflecting quality of care should be collected in a systemic manner, have means of statistical analysis of results, and develop an approach to problem solving that involves feedback on the effectiveness of solutions. The SVIN SILC Writing Committee strongly encourages all stroke interventional laboratories to participate in a national registry and help develop quality benchmarks to provide ongoing system for tracking complications and outcomes. Time-based metrics for efficiency of the stroke interventional laboratory (such as 'door to groin', 'door to perfusion', etc.), as well as the revascularization metrics for assessing the effectiveness of stroke interventions performed (such as 'thrombolysis in cerebral ischemic - TICI scores', etc.) need to be collected and analyzed. These stroke interventional laboratories for brain attack care are similar to PCI-capable labs for heart attack care.

Facilities that do not intend to or do not have the resources to provide $24 / 7$ stroke interventional services, may choose to provide diagnostic cerebral angiography services for stroke care. These cerebral angiography laboratories for brain attack care are similar to cardiac catheterization laboratories for heart attack care, and shall strictly adhere to diagnosis of cerebrovascular disorders. These cerebral angiography laboratories may or may not have neurosurgical services and advanced support services such as neurocritical care, renal with hemodialysis, and on call anesthesiology services available, since the complication rates of diagnostic services are low. Physicians at these cerebral angiography laboratories must have admission privileges in case of complications. Cerebral angiography volumes must be $>100$ per year [45].

Fully certified stroke interventional laboratories, with full support services listed above readily available, may perform stroke interventions in addition to cerebral angiograms (table 4). These stroke interventional laboratories are ideally associated with a compre- 
Table 4. Optimal clinical and support services for a successful stroke interventional lab

\section{DOI: $10.1159 / 000443617$ \\ c) 2016 S. Karger AG, Basel www.karger.com/ine \\ Shams et al.: Society of Vascular and Interventional Neurology (SVIN) Stroke} Interventional Laboratory Consensus (SILC) Criteria

hensive stroke center but may be associated with a primary stroke center that has the necessary support services listed above, and may be affiliated with an academic or communitybased practice. Based on the current requirements of TJC, DNV, and HFAP, the SVIN SILC Writing Committee recommends that adequate volumes must be maintained at these stroke interventional laboratories for providing high-quality stroke care, with a minimum requirement of $>100$ diagnostic cerebral angiograms per year and greater than 30 stroke interventions for LVO AIS (mechanical thrombectomies) per year [48].

\section{Section 7: Metrics (Safety) - Radiation and Procedural Safety Practices}

Standard 7.1 - Radiation Safety

All supervising physicians, registered radiology assistants, and radiological technologists are responsible for safety in the workplace by keeping radiation exposure of staff and patient. Operators are to practice the ALARA method (As Low As Reasonably Achievable) in order to safely assure radiation doses to individual patients are appropriate and minimize the risk of radiation exposure while maintaining adequate image integrity for diagnosis and management of cerebrovascular disease states $[52,73]$. All personnel and staff working in the stroke interventional laboratory must understand the key principles of occupational and public radiation protection and proper management of radiation dose to patients. The SVIN SILC Writing Committee recommends that the minimum requirements of annual continuing medical education courses for radiation safety for all staff and physicians within a stroke interventional laboratory as well as compliance with any state or national radiation safety requirements are met. Radiation safety officer must assist all laboratory staff in policies and procedures for safe handling of radiopharmaceuticals in compliance with the ALARA guidelines. All policies and procedures must further be in compliance with any radiation safety regulatory body including Nuclear Regulatory Commission and/or any local regulatory agencies. Radiopharmaceutical doses should be tailored for individual patient by protocol.

Radiation exposure must be measured by single device monitoring or the two-monitor technique with either X-ray film badges or transluminescent dosimeter badges on a continual basis. Dosage levels per procedure must be documented and maintained as part of patient medical records for at least 7 years or according to local medical records standards. Operators should always practice standard of care with appropriate shielding and adequate distance. Pregnant workers may continue to work in the stroke interventional laboratory if they choose. Fetal exposure, as measured by a waist dosimeter, should be no more than 0.05 $\mathrm{rem} / \mathrm{month}$, or $<0.5 \mathrm{rem}$ for the entire pregnancy, and as per each state or national regulatory and radiation safety requirements. 
Fig. 6. Average radiation doses of stroke interventional procedures over a 12-month period at a comprehensive stroke center.

\section{DOI: $10.1159 / 000443617$}

Shams et al:: Society of Vascular and Interventional Neurology (SVIN) Stroke Interventional Laboratory Consensus (SILC) Criteria

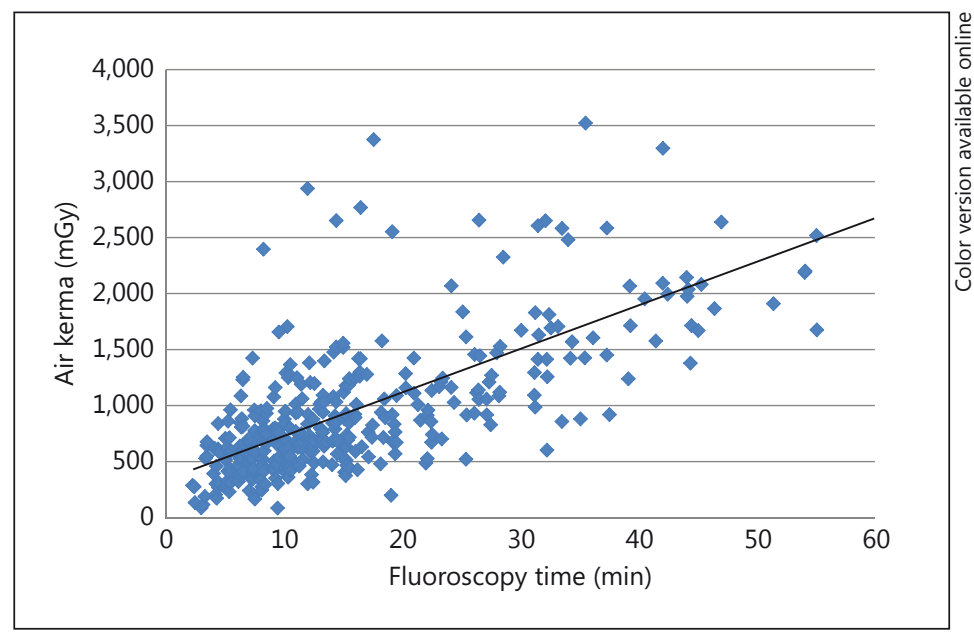

Standard 7.2 - Ionic Contrast Safety

Adverse reactions to contrast agents may range from mild allergic reactions to anaphylaxis, renal toxicity, and life-threatening emergency. Factors associated with adverse reactions are history of previous allergy to contrast material, asthma, and other allergies. Prescreening and prophylactic treatments before contrast administration can prevent potential reactions. Ionic contrast material is usually higher in osmolar content compared to nonionic contrast materials, and generally more toxic. All contrast materials should be used at the lowest possible dose per procedure per patient. Physicians and staff must be aware of such complications to ensure prompt diagnosis and treatment.

Standard 7.3 - Acceptable Fluoroscopic Time for Diagnostic Cerebral Angiography and

Stroke Interventional Procedures

Four radiation dose metrics should be kept in mind and monitored throughout every stroke interventional procedure: reference air kerma in milligrays, kerma-area product in centigray-square centimeters, fluoroscopy time, and number of fluoroscopic images. Fluoroscopy time and, more specifically, reference air kerma and kerma-area product are good tools to estimate the risk of radiation injury. Average doses of stroke interventions nationally by procedure are currently unknown (fig. 6).

Reference levels are a guide to good practice, but they are neither dose limits nor threshold levels that define competent performance of the operator or the equipment. If the radiation dose for a specific case or the mean dose for a number of cases of a procedure exceeds the reference level, it does not mean that the procedure or procedures have been performed improperly. Similarly, a mean dose for a procedure that is less than the reference level does not guarantee that the procedure is being performed optimally. All certified stroke interventional procedures must document the above parameters, and a task force must review outliers at regular intervals.

\section{Discussion}

Heart attack care for STEMIs has evolved over the last two decades with the advent of PCIs with balloons and stent [74]. EMS triage for heart attacks has been simplified with the use of EKG, and patients with STEMIs are triaged to a PCI-capable lab (device therapy), and those 
without STEMIs are triaged to a non-PCI facility (drug therapy) [74]. Standardized consensus criteria for PCI-capable labs and cardiac catheterization labs have improved access to care and outcomes [74]. Similarly, brain attack care for LVO's has recently evolved with the completion of several landmark trials in favor of stroke interventions (aka endovascular stroke therapy) for LVOs [2]. EMS triage for brain attacks can be simplified similar to heart attacks with the use of stroke severity assessments by EMS en route to a facility [75]. Those patients with high likelihood of an LVO can be triaged to a stroke interventional laboratory capable (SILC) facility (drug plus device therapy; IV t-PA plus mechanical thrombectomy), and those with low likelihood of an LVO can be triaged to a non-SILC facility (drug therapy; IV t-PA). Stroke intervention is a rapidly evolving field with an exponential potential to impact reduction of mortality and morbidity rates by organizing stroke systems of care, with appropriate EMS triage, and standardized stroke interventional laboratories with the highest quality standards.

As we encounter paradigm shifts in stroke treatments, with accumulating evidence through randomized controlled trials for increasing benefits of stroke interventional treatments for LVO AIS [2], the stroke interventional community ought to initiate similar organization models and levels of responsibilities of the stroke interventional laboratories. In the absence of benchmarks, organization, and timely delivery of care, stroke interventionalists risk compromising quality of care that can directly impact short and long-term outcomes. Standardization of SILC criteria is designed to improve stroke and cerebrovascular disease outcomes with appropriate oversight of personnel and logistic components of a stroke interventional laboratory.

The 7Ms, manpower, materials, methods, metrics (volume), metrics (quality), and metrics (safety), in the lean operations of a system of stroke care are each factors that contribute towards a standardized stroke interventional laboratory with quality assurance and continued improvement in optimal stroke care. Adoption of such a system will identify value-added services, wasteful services and time delays, and identify missed opportunities for further improvements.

The SVIN Writing committee endorses appropriate training standards and enforcing existing requirements as well as monitoring total volume of each facility and operator $[43,45$, 47]. Quality metrics, time-based metrics and task forces, as well as direct oversight by stroke interventional physicians are essential to improve outcomes. Lastly, stroke intervention provides a unique treatment to the community with dire unmet needs.

\section{Lean Supply Operations and Brain Attack Care: Look towards the Future}

The SILC criteria further address the importance of ultra-early delivery of care within the first $60 \mathrm{~min}$ from the onset of symptoms, termed as the golden hour of stroke. European countries including Germany, UK, Spain, and Netherlands have succeeded in decreasing presentation times (first medical contact to door) as well as implementing key management steps of stroke code in the field en route to a facility. EMS triage for brain attacks using stroke severity assessments (such as LAMS, LEGS, RACE, 3ISS, NIHSS etc.) will help optimize prehospital workflow, and increase the likelihood of LVOs being triaged to a stroke interventional laboratory for early revascularization [75-79]. By uniformly organizing prehospital, ED, and stroke interventional laboratory management into a single supply chain, the stroke community will surely succeed in providing every stroke patient in the country with timely, appropriate treatments.

\section{Disclosure Statement}

Dileep Yavagal is a consultant to Medtronic, steering committee member of Swift Prime trial, and past president of SVIN. Tudor Jovin has consulted for Codman Neurovascular and Neuravi, holds stock in Silk Road and Blockade, has acted as an unpaid consultant to Stryker as PI of the DAWN trial, and served as an unpaid member of a Medtronic Advisory Board. Vallabh Janardhan is a board member of Insera Therapeutics Inc. and SVIN. Tanzila Shams, Osama Zaidat, and Andrew Xavier have no financial disclosures. 


\begin{tabular}{l|l}
\hline \multicolumn{2}{l}{ Intervent Neurol 2016;5:1-28 } \\
\hline DOI: $10.1159 / 000443617$ & $\begin{array}{l}\text { C 2016 S. Karger AG, Basel } \\
\text { www.karger.com/ine }\end{array}$ \\
\hline
\end{tabular}

Shams et al.: Society of Vascular and Interventional Neurology (SVIN) Stroke Interventional Laboratory Consensus (SILC) Criteria

\section{References}

1 Janardhan V, Gianatasio R, Chen S, Bhuva P, Murray M, Santos P, et al: Preliminary results from the FIRST trial: natural history of acute stroke from large vessel occlusion (abstract 194). Int Stroke Conf, Los Angeles, February 2013.

-2 Ding D: Endovascular mechanical thrombectomy for acute ischemic stroke: a new standard of care. J Stroke 2015;17:123.

-3 Manjila S, Masri T, Shams T, Chowdhry SA, Sila C, Selman WR: Evidence-based review of primary and secondary ischemic stroke prevention in adults: a neurosurgical perspective. Neurosurg Focus 2011;30:E1.

4 Badhiwala JH, Nassiri F, Alhazzani W, Selim MH, Farrokhyar F, Spears J, et al: Endovascular thrombectomy for acute ischemic stroke: a meta-analysis. JAMA 2015;314:1832-1843.

5 Schwamm LH, Pancioli A, Acker JE, Goldstein LB, Zorowitz RD, Shephard TJ, et al: Recommendations for the establishment of stroke systems of care: recommendations from the American Stroke Association's Task Force on the Development of Stroke Systems. Circulation 2005;111:1078-1091.

-6 Leifer D, Bravata DM, Connors JJB, Hinchey JA, Jauch EC, Johnston SC, et al: Metrics for measuring quality of care in comprehensive stroke centers: detailed follow-up to Brain Attack Coalition comprehensive stroke center recommendations: a statement for healthcare professionals from the American Heart Association/ American Stroke Association. Stroke J Cereb Circ 2011;42:849-877.

7 Healthcare Facilities Accreditation Program (HFAP) - The Certification Process. http://www.hfap.org/CertificationPrograms/certificationProcess.aspx (cited January 23, 2015).

-8 Grigoryan M, Chaudhry SA, Hassan AE, Suri FK, Qureshi AI: Neurointerventional procedural volume per hospital in United States: implications for comprehensive stroke center designation. Stroke J Cereb Circ 2012; 43:1309-1314.

-9 Qureshi AI, Chaudhry SA, Majidi S, Grigoryan M, Rodriguez GJ, Suri MFK: Population-based estimates of neuroendovascular procedures: results of a state-wide study. Neuroepidemiology 2012;39:125-130.

10 Warach S: Prehospital thrombolysis for stroke: an idea whose golden hour has arrived. JAMA Neurol 2015;72: 9.

11 CDC - Access to Trauma Care - Trauma Care - Injury. http://www.cdc.gov/traumacare/access_trauma.html (cited February 8, 2015).

12 Bazzoli GJ, MacKenzie EJ: Trauma centers in the United States: identification and examination of key characteristics. J Trauma 1995;38:103-110.

13 TraumaCenter LevelsExplained-AmericanTraumaSociety.http://www.amtrauma.org/?page=TraumaLevels (cited January 23, 2015).

14 Fassbender K, Balucani C, WalterS, LevineSR, Haass A, Grotta J: Streamlining of prehospital stroke management: the golden hour. Lancet Neurol 2013;12:585-596.

15 American Heart Association, Mission Lifeline: STEMI Systems of Care. http://www.heart.org/HEARTORG/ HealthcareResearch/MissionLifelineHomePage/LearnAboutMissionLifeline/STEMI-Systems-of-Care_ UCM_439065_SubHomePage.jsp (cited February 7, 2015).

16 Waters RE, Singh KP, Roe MT, Lotfi M, Sketch MH, Mahaffey KW, et al: Rationale and strategies for implementing community-based transfer protocols for primary percutaneous coronary intervention for acute ST-segment elevation myocardial infarction. J Am Coll Cardiol 2004;43:2153-2159.

17 Bagai A, Al-Khalidi HR, Sherwood MW, Muñoz D, Roettig ML, Jollis JG, et al: Regional systems of care demonstration project: Mission: Lifeline STEMI Systems Accelerator: design and methodology. Am Heart J 2014;167: 15-21.e3.

18 Thomas JL, French WJ: Current state of ST-segment myocardial infarction: evidence-based therapies and optimal patient outcomes in advanced systems of care. Cardiol Clin 2014;32:371-385.

19 Fonarow GC, Smith EE, Saver JL, Reeves MJ, Hernandez AF, Peterson ED, et al: Improving door-to-needle times in acute ischemic stroke: the design and rationale for the American Heart Association/American Stroke Association's Target: Stroke Initiative. Stroke 2011;42:2983-2989.

-20 Fransen PSS, Beumer D, Berkhemer OA, van den Berg LA, Lingsma H, van der Lugt A, et al: MR CLEAN, a multicenter randomized clinical trial of endovascular treatment for acute ischemic stroke in the Netherlands: study protocol for a randomized controlled trial. Trials 2014;15:343.

-21 Demchuk AM, Goyal M, Menon BK, Eesa M, Ryckborst KJ, Kamal N, et al: Endovascular treatment for Small Core and Anterior circulation Proximal occlusion with Emphasis on minimizing CT to recanalization times (ESCAPE) trial: methodology. Int J Stroke 2015;10:429-438.

-22 Campbell BCV, Mitchell PJ, Yan B, Parsons MW, Christensen S, Churilov L, et al: A multicenter, randomized, controlled study to investigate EXtending the time for Thrombolysis in Emergency Neurological Deficits with Intra-Arterial therapy (EXTEND-IA). Int J Stroke 2014;9:126-132.

-23 Saver JL, Goyal M, Bonafe A, Diener H-C, Levy EI, Pereira VM, et al: Solitaire ${ }^{\mathrm{TM}}$ with the Intention for Thrombectomy as Primary Endovascular Treatment for Acute Ischemic Stroke (SWIFT PRIME) trial: protocol for a randomized, controlled, multicenter study comparing the Solitaire revascularization device with IV tPA with IV tPA alone in acute ischemic stroke. Int J Stroke Off J Int Stroke Soc 2015;10:439-448.

-24 Eesa M, Menon BK, Hill MD, Demchuk A, Goyal M: Achieving faster recanalization times by IA thrombolysis in acute ischemic stroke: where should we direct our efforts? Interv Neuroradiol 2011;17:228-234. 
Shams et al:: Society of Vascular and Interventional Neurology (SVIN) Stroke Interventional Laboratory Consensus (SILC) Criteria

25 Asimos AW, Ward S, Brice JH, Rosamond WD, Goldstein LB, Studnek J: Out-of-hospital stroke screen accuracy in a state with an emergency medical services protocol for routing patients to acute stroke centers. Ann Emerg Med 2014;64:509-515.

26 Kothari R, Barsan W, Brott T, Broderick J, Ashbrock S: Frequency and accuracy of prehospital diagnosis of acute stroke. Stroke J Cereb Circ 1995;26:937-941.

27 Ragoschke-Schumm A, Walter S, Haass A, Balucani C, Lesmeister M, Nasreldein A, et al: Translation of the 'time is brain' concept into clinical practice: focus on prehospital stroke management. Int J Stroke 2014;9:333-340.

28 Saver JL, Starkman S, Eckstein M, Stratton S, Pratt F, Hamilton S, et al: Methodology of the Field Administration of Stroke Therapy - Magnesium (FAST-MAG) phase 3 trial: Part 1 - rationale and general methods. Int J Stroke 2014;9:215-219.

29 Hatez S: The beneficial failure of the FAST-MAG trial. Prehospital magnesium didn't help stroke patients, but we still learned a lot. EMS World 2014;43:58-60.

30 Walter S, Kostopoulos P, Haass A, Keller I, Lesmeister M, Schlechtriemen T, et al: Diagnosis and treatment of patients with stroke in a mobile stroke unit versus in hospital: a randomised controlled trial. Lancet Neurol 2012;11:397-404.

-31 Weber JE, Ebinger M, Rozanski M, Waldschmidt C, Wendt M, Winter B, et al: Prehospital thrombolysis in acute stroke: results of the PHANTOM-S pilot study. Neurology 2013;80:163-168.

-32 Sacks D, Black CM, Cognard C, Connors JJ, Frei D, Gupta R, et al: Multisociety consensus quality improvement guidelines for intraarterial catheter-directed treatment of acute ischemic stroke, from the American Society of Neuroradiology, Canadian Interventional Radiology Association, Cardiovascular and Interventional Rad: Multisociety QI Guidelines for Intraarterial CDT for Stroke. Catheter Cardiovasc Interv 2013;82:E52-E68.

-33 Connors JJ, Sacks D, Furlan AJ, Selman WR, Russell EJ, Stieg PE, et al: Training, competency, and credentialing standards for diagnostic cervicocerebral angiography, carotid stenting, and cerebrovascular intervention: a joint statement from the American Academy of Neurology, the American Association of Neurological Surgeons, the American Society of Interventional and Therapeutic Neuroradiology, the American Society of Neuroradiology, the Congress of Neurological Surgeons, the AANS/CNS Cerebrovascular Section, and the Society of Interventional Radiology. J Vasc Interv Radiol 2004;15:1347-1356.

-34 Heiserman JE, Dean BL, Hodak JA, Flom RA, Bird CR, Drayer BP, et al: Neurologic complications of cerebral angiography. AJNR Am J Neuroradiol 1994;15:1401-1407; discussion 1408-1411.

-35 Leffers AM, Wagner A: Neurologic complications of cerebral angiography. A retrospective study of complication rate and patient risk factors. Acta Radiol 2000;41:204-210.

-36 Sassi M, Servello D, Porta M: The impact of technological advances in neurosurgery: historical perspectives and new developments. Funct Neurol 2009;24:221-224.

-37 Loose R, Braunschweig R, Kotter E, Mildenberger P, Simmler R, Wucherer M: Compression of digital images in radiology - results of a consensus conference (in German). RoFo 2009;181:32-37.

-38 Ellis MJ, Amlie-Lefond C, Orbach DB: Endovascular therapy in children with acute ischemic stroke: review and recommendations. Neurology 2012;79(suppl 1):S158-S164.

39 De Keyser J, Gdovinová Z, Uyttenboogaart M, Vroomen PC, Luijckx GJ: Intravenous alteplase for stroke: beyond the guidelines and in particular clinical situations. Stroke J Cereb Circ 2007;38:2612-2618.

-40 Dagi TF: Neurosurgery and the introduction of cerebral angiography. Neurosurg Clin North Am 2001;12: 145-153, ix.

41 Ligon BL: The mystery of angiography and the 'unawarded' Nobel Prize: Egas Moniz and Hans Christian Jacobaeus. Neurosurgery 1998;43:602-611.

42 Hinck VC, Judkins MP, Paxton HD: Simplified selective femorocerebral angiography. Radiology 1967;89:10481052.

-43 Garg A: E-071 organization of a neurointerventional fellowship curriculum. J Neurointervent Surg 2014; 6(suppl 1):A71-A72.

-44 Meyers PM, Schumacher HC, Alexander MJ, Derdeyn CP, Furlan AJ, Higashida RT, et al: Performance and training standards for endovascular acute ischemic stroke treatment. Neurology 2012;79(suppl 1):S234S238.

45 Qureshi AI, Abou-Chebl A, Jovin TG: Qualification requirements for performing neurointerventional procedures: a Report of the Practice Guidelines Committee of the American Society of Neuroimaging and the Society of Vascular and Interventional Neurology. J Neuroimaging 2008;18:433-447.

46 Go AS, Mozaffarian D, Roger VL, Benjamin EJ, Berry JD, Blaha MJ, et al: Executive summary: heart disease and stroke statistics - 2014 update: a report from the American Heart Association. Circulation 2014;129:399-410.

-47 Qureshi AI, Suri MF, Khan J, Kim SH, Fessler RD, Ringer AJ, et al: Endovascular treatment of intracranial aneurysms by using Guglielmi detachable coils in awake patients: safety and feasibility. J Neurosurg 2001;94: 880-885.

48 Barr JD, Connors JJ, Sacks D, Wojak JC, Becker GJ, Cardella JF, et al: Quality improvement guidelines for the performance of cervical carotid angioplasty and stent placement. J Vasc Interv Radiol 2003;14:S321-S335.

-49 Hashimoto T, Gupta DK, Young WL: Interventional neuroradiology - anesthetic considerations. Anesthesiol Clin North Am 2002;20:347-359, vi.

50 Abou-Chebl A, Lin R, Hussain MS, Jovin TG, Levy EI, Liebeskind DS, et al: Conscious sedation versus general anesthesia during endovascular therapy for acute anterior circulation stroke: preliminary results from a retrospective, multicenter study. Stroke J Cereb Circ 2010;41:1175-1179. 


\begin{tabular}{l|l}
\hline \multicolumn{2}{l}{ Intervent Neurol 2016;5:1-28 } \\
\hline DOI: $10.1159 / 000443617$ & $\begin{array}{l}\text { C 2016 S. Karger AG, Basel } \\
\text { www.karger.com/ine }\end{array}$ \\
\hline
\end{tabular}

Shams et al.: Society of Vascular and Interventional Neurology (SVIN) Stroke Interventional Laboratory Consensus (SILC) Criteria

51 McDonagh DL, Olson DM, Kalia JS, Gupta R, Abou-Chebl A, Zaidat 00: Anesthesia and sedation practices among neurointerventionalists during acute ischemic stroke endovascular therapy. Front Neurol 2010;1:118.

52 Cardella JF, Casarella WJ, DeWeese JA, Dorros GM, et al: Optimal resources for the examination and endovascular treatment of the peripheral and visceral vascular systems. AHA Intercouncil Report on Peripheral and Visceral Angiographic and Interventional Laboratories. J Vasc Interv Radiol 2003;14(9 Pt 2):S517-S530.

-53 Zoetelief J, Faulkner K: Equipment requirements and specification for digital and interventional radiology. Radiat Prot Dosimetry 2001;94:43-48.

54 American Society of Interventional and Therapeutic Neuroradiology: General considerations for endovascular surgical neuroradiologic procedures. AJNR Am J Neuroradiol 2001;22(suppl):S1-S3.

-55 Swetadri Vasan SN, Ionita C, Bednarek DR, Rudin S: A novel Region of Interest (ROI) imaging technique for biplane imaging in interventional suites: high-resolution small field-of-view imaging in the frontal plane and dose-reduced, large field-of-view standard-resolution imaging in the lateral plane. Proc SPIE Int Soc Opt Eng 2014;9033:90332F.

56 Shah S, Murthy SB, Hannawi Y, Rao CPV: The utility of cone beam volume CT in the evaluation of thrombosed intracranial aneurysms in subarachnoid hemorrhage. J Neurointervent Surg 2013;5:e51.

57 Broderick JP, Lu M, Kothari R, Levine SR, Lyden PD, Haley EC, et al: Finding the most powerful measures of the effectiveness of tissue plasminogen activator in the NINDS tPA stroke trial. Stroke J Cereb Circ 2000;31:23352341.

58 Hacke W, Donnan G, Fieschi C, Kaste M, von Kummer R, Broderick JP, et al: Association of outcome with early stroke treatment: pooled analysis of ATLANTIS, ECASS, and NINDS rt-PA stroke trials. Lancet 2004;363:768774.

59 Broderick JP, Palesch YY, Demchuk AM, Yeatts SD, Khatri P, Hill MD, et al: Evolution of practice during the Interventional Management of Stroke III Trial and implications for ongoing trials. Stroke 2014;45:3606-3611.

60 Janardhan V, Yoo AJ, Frei D, Ammar L, Kuo SS, Udea D, et al: Significant correlation between neurological and functional recoveries in the natural history of acute ischemic stroke from large vessel occlusion. Stroke 2015; 46(suppl 1):AWP14.

61 Simonsen S, Andresen M, Michelsen L, Viereck S, Lippert FK, Iversen H: Evaluation of pre-hospital transport time of stroke patients to thrombolytic treatment. Scand J Trauma Resusc Emerg Med 2014;22:65.

62 Lazzaro MA, Novakovic RL, Alexandrov AV, Darkhabani Z, Edgell RC, English J, et al: Developing practice recommendations for endovascular revascularization for acute ischemic stroke. Neurology 2012;79(suppl 1):S243-S255.

63 Wen C-S, Lin S-M, Chen Y, Chen J-C, Wang Y-H, Tseng S-H: Radiation-induced temporary alopecia after embolization of cerebral arteriovenous malformations. Clin Neurol Neurosurg 2003;105:215-217.

-64 Podlipnik S, Giavedoni P, San-Román L, Ferrando J: Square alopecia: a new type of transient alopecia of the scalp following fluoroscopically endovascular embolization. Int J Trichology 2013;5:201.

-65 DeBaun MR, Gordon M, McKinstry RC, Noetzel MJ, White DA, Sarnaik SA, et al: Controlled trial of transfusions for silent cerebral infarcts in sickle cell anemia. N Engl J Med 2014;371:699-710.

66 Brott TG, Halperin JL, Abbara S, Bacharach JM, Barr JD, Bush RL, et al: 2011 ASA/ACCF/AHA/AANN/AANS/ ACR/ASNR/CNS/SAIP/SCAI/SIR/SNIS/SVM/SVS guideline on the management of patients with extracranial carotid and vertebral artery disease: executive summary: a report of the American College of Cardiology Foundation/American Heart Association Task Force on Practice Guidelines, and the American Stroke Association, American Association of Neuroscience Nurses, American Association of Neurological Surgeons, American College of Radiology, American Society of Neuroradiology, Congress of Neurological Surgeons, Society of Atherosclerosis Imaging and Prevention, Society for Cardiovascular Angiography and Interventions, Society of Interventional Radiology, Society of NeuroInterventional Surgery, Society for Vascular Medicine, and Society for Vascular Surgery. Vasc Med Lond Engl 2011;16:35-77.

67 Qureshi AI, Janardhan V, Memon MZ, Suri MFK, Shah QA, Miley JT, et al: Initial experience in establishing an academic neuroendovascular service: program building, procedural types, and outcomes. J Neuroimaging 2009;19:72-79.

68 Internet Stroke Center. http://www.strokecenter.org/ (cited December 9, 2014).

-69 Chaturvedi S, Dumont AS: The learning curve for neuroendovascular procedures: how important is it? Neurology 2009;72:1974-1975.

-70 Bates ER, Babb JD, Casey DE, Cates CU, Duckwiler GR, Feldman TE, et al: ACCF/SCAI/SVMB/SIR/ASITN 2007 Clinical Expert Consensus Document on Carotid Stenting. J Am Coll Cardiol 2007;49:126-170.

71 Molyneux AJ, Kerr RSC, Yu L-M, Clarke M, Sneade M, Yarnold JA, et al: International subarachnoid aneurysm trial (ISAT) of neurosurgical clipping versus endovascular coiling in 2,143 patients with ruptured intracranial aneurysms: a randomised comparison of effects on survival, dependency, seizures, rebleeding, subgroups, and aneurysm occlusion. Lancet 2005;366:809-817.

-72 Rutledge WC, Abla AA, Nelson J, Halbach VV, Kim H, Lawton MT: Treatment and outcomes of ARUBA-eligible patients with unruptured brain arteriovenous malformations at a single institution. Neurosurg Focus 2014; 37:E8.

73 Oostema JA, Nasiri M, Chassee T, Reeves MJ: The quality of prehospital ischemic stroke care: compliance with guidelines and impact on in-hospital stroke response. J Stroke Cerebrovasc Dis 2014;23:2773-2779. 
74 Natarajan MK, Paul N, Mercuri M, Waller EJ, Leipsic J, Traboulsi M, et al: Canadian Cardiovascular Society position statement on radiation exposure from cardiac imaging and interventional procedures. Can J Cardiol 2013;29:1361-1368.

75 Venizelos A, Chen SH, Gianatasio RM, Coffman SR, Gamber M, Janardhan V: Texas Stroke Intervention PreHospital Stroke Severity Scale (aka LEGS score): a triaging tool for interventional stroke therapy. Stroke 2014; 45:ATMP59.

76 Bashore TM, Bates ER, Berger PB, Clark DA, Cusma JT, Dehmer GJ, et al: American College of Cardiology/ Society for Cardiac Angiography and Interventions Clinical Expert Consensus Document on cardiac catheterization laboratory standards. A report of the American College of Cardiology Task Force on Clinical Expert Consensus Documents. J Am Coll Cardiol 2001;37:2170-2214.

77 Nazliel B, Starkman S, Liebeskind DS, Ovbiagele B, Kim D, Sanossian N, et al: A brief prehospital stroke severity scale identifies ischemic stroke patients harboring persisting large arterial occlusions. Stroke J Cereb Circ 2008;39:2264-2267.

-78 Singer OC, Dvorak F, du Mesnil de Rochemont R, Lanfermann H, Sitzer M, Neumann-Haefelin T: A simple 3-item stroke scale: comparison with the National Institutes of Health Stroke Scale and prediction of middle cerebral artery occlusion. Stroke J Cereb Circ 2005;36:773-776.

79 Pérez de la Ossa N, Carrera D, Gorchs M, Querol M, Millán M, Gomis M, et al: Design and validation of a prehospital stroke scale to predict large arterial occlusion: the rapid arterial occlusion evaluation scale. Stroke J Cereb Circ 2014;45:87-91.

80 ACGME Program Requirements for Graduate Medical Education in Endovascular Surgical Neuroradiology (Diagnostic Radiology, Neurological Surgery, or Neurology). 2007. http://www.acgme.org/acgmeweb/ Portals/0/PFAssets/ProgramRequirements/163-182-422_endovascular_neuroradiology_2016_1-YR.pdf.

-81 Rosenfield KM; CAI/SVMB/SVS Writing Committee: Clinical competence statement on carotid stenting: training and credentialing for carotid stenting - multispecialty consensus recommendations. J Vasc Surg 2005; 41:160-168.

-82 IMS Study Investigators: Combined intravenous and intra-arterial recanalization for acute ischemic stroke: the Interventional Management of Stroke Study. Stroke J Cereb Circ 2004;35:904-911. 


\section{Erratum}

In the article by Shams et al., entitled 'Society of Vascular and Interventional Neurology (SVIN) Stroke Interventional Laboratory Consensus (SILC) Criteria: a 7M management approach to developing a stroke interventional laboratory in the era of stroke thrombectomy for large vessel occlusions' [Intervent Neurol 2016;5:1-28, DOI: 10.1159/000443617], in the reference list, the following reference should be inserted as the new number 24: Jovin T, et al: Thrombectomy within 8 hours after symptom onset in ischemic stroke. $\mathrm{N}$ Engl J Med 2015;372:2296-2306. The subsequent references should be renumbered accordingly. The current numbers 45 and 46, however, should swap places and become 47 and 46.

In the text, current reference [10-12] on page 4 should become [10-13], and as a consequence, the subsequent references should be renumbered accordingly, adding 1 . The current $[15,16]$ should become $[16-18]$, [17] should become [19] and [18] should also become [19]. The following reference [23] on page 5 should remain, but the subsequent references again should be renumbered, adding 1. Reference [31] on page 6 should become [32, 33], the subsequent references should be renumbered, adding 1, which also should include the references in table 2 . On page 16 , however, the current reference $[2,19]$ should become $[2$, $20-24]$, and on page 19 , the current reference $[7,68]$ should remain the same.

Furthermore, in figure 1, a last line reading '2015 - REVASCAT - 0-8 hrs IA endovascular clot retrieval with Stentriever' should be inserted. As a general rule, in the third column, 'Penumbra' should be replaced by 'Thromboaspiration' and 'Solitaire FR' as well as 'Trevo' should be replaced by 'Stentriever'.

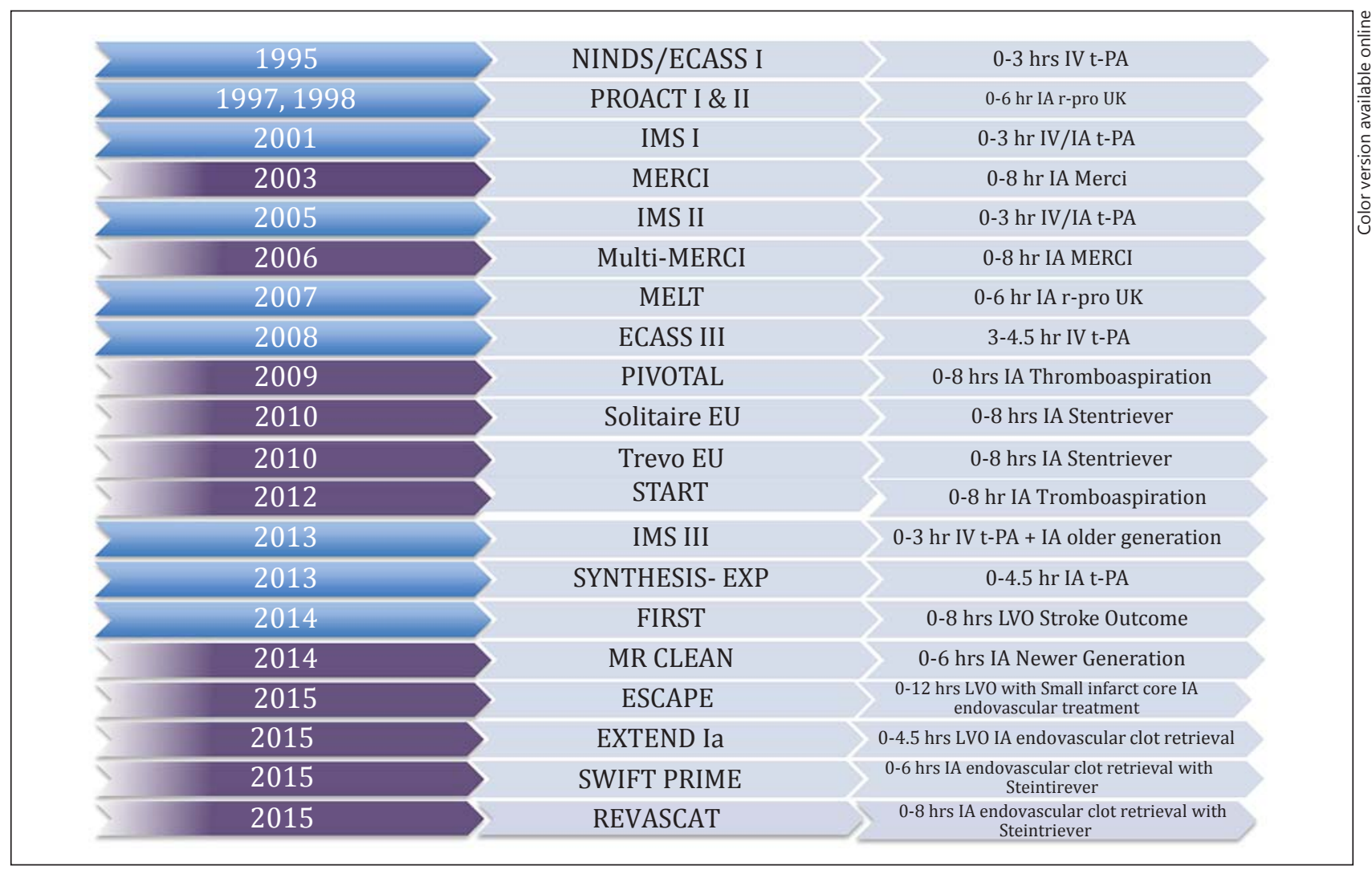

Fig. 1. Landmark stroke interventional trials. IA = Intra-arterial. Blue color indicates MEDICAL therapeutics trials. Purple color indicates device-based stroke interventional trials. Colors refer to the online version only. 